\title{
Experimental Study on the Electromechanical Hysteresis Property of Macro Fibre Composite Actuator
}

\author{
Chen Luqi \\ Department of Civil Engineering, Xi'an Jiaotong University, Xi'an, 710049, China. \\ State Key Laboratory for Mechanical Behavior of Materials, Xi'an Jiaotong University, Xi'an, 710049, China.
}

\author{
Wu Xiaohong \\ School of Aerospace, Xi'an Jiaotong University, Xi'an, China.
}

\author{
Sun Qing and Xue Xiaomin \\ Department of Civil Engineering, Xi'an Jiaotong University, Xi'an, 710049, China.
}

(Received 28 January 2015; accepted 13 April 2016)

The hysteresis characteristic in macro fibre composite (MFC) actuator is intimately related to their application in vibration control system. In this paper, the electromechanical hysteresis property of MFC actuator is studied. First, experimental study on the electromechanical behaviours under different voltages and frequencies is carried out, and the hysteretic property of MFC actuator is investigated. A digital signal processing (DSP) system is used to control input voltage and a digital image correlation (DIC) system as noncontact setup is used to obtain the output strain of the MFC actuator in the experiment. The experimental results indicate that the relationship of the voltage and strain displays hysteresis with nonlocal memory. Second, the Preisach model is used to describe hysteresis characteristic of the MFC actuator. In order to improve the accuracy of the model, the modifications are made, in which the experimental data under the quasi-static frequency range and the congruency property are used to establish the modified Preisach model. Finally, the hysteresis characteristics of the MFC actuator predicted from the proposed model are compared with those obtained from the classical Preisach model. The results indicate that the proposed model gives better accuracy than the classical Preisach model, and it is suggested that the present study on the hysteresis model of the MFC actuator can be used in the active vibration control.

\section{INTRODUCTION}

Macro fibre composite (MFC) actuators are known to possess several salient properties such as durability, surfacecomfortability, and higher ability to eliminate vibration in comparison with the conventional piezoceramics actuators. ${ }^{1,2}$ Hence, it is widely recognized to play an essential role in applications ranging from vibration control, ${ }^{3-6}$ structural health monitoring, ${ }^{7,8}$ and energy harvesting. ${ }^{9,10}$

However, hysteresis behaviour is a major obstacle in application of MFC actuators. Like many other piezoelectric materials, the MFC actuator exhibits hysteresis property with nonlocal memory, in which the response depends on both the previous extremums as well as the present value of input voltage. ${ }^{11}$ If the hysteresis behaviour is not taken into account, the predicted response of the MFC actuator will significantly deviate from accuracy. Therefore, in order to successfully apply the MFC actuator in structural active vibration control, it is necessary to establish a hysteresis model to describe electromechanical behaviour of the MFC actuator.

In the previous study, the approaches of modelling the hysteresis behaviour of piezoelectric actuator are divided into two groups: physical model and phenomenological model. ${ }^{12}$ The former models, like Jiles-Atherton mode $1^{13}$ and homogenized energy model, ${ }^{14}$ are formulated based on the physical principle of domain wall movements and internal energy losses in the material. Nevertheless, these physical models are difficult to be applied in control system due to the complex physical cause of hysteresis phenomenon of piezoelectric actuator. The phenomenological models are formulated based on a purely mathematical expression that relates the overall inputoutput relationship without considering the underlying physical essence. Several examples of such models are the generalized Maxwell model, ${ }^{15,16}$ the Prandtl-Ishlinskii model, ${ }^{17}$ and the Preisach model. ${ }^{18-26}$ However, the symmetric structures of the generalized Maxwell and the Prandtl-Ishlinskii model limit their accuracy. The Preisach model, which was introduced by Preisach, ${ }^{18}$ is a phenomenological hysteresis model. Although it originated in magnetics, the model has been utilized in many other areas later, such as superconductors, electro rheological fluids, shape memory alloys, and piezoelectric materials. ${ }^{19}$ Hughes and Wen ${ }^{20}$ discussed the unified framework provided by the classical Preisach model for both piezoceramic and shape memory alloy. The identification method based on the input/output data and hysteresis compensation using the classical Preisach model was also demonstrated in their research. Based on the previous research, Ge and Jouaneh ${ }^{21,22}$ and Robert et al. ${ }^{23,24}$ developed a tracking control approach for piezoceramic actuator by incorporating the classical Preisach model in the feed-forward loop.

Since the MFC actuator was produced by Smart-Material Corporation ${ }^{25}$ researchers extensively studied the hysteresis 


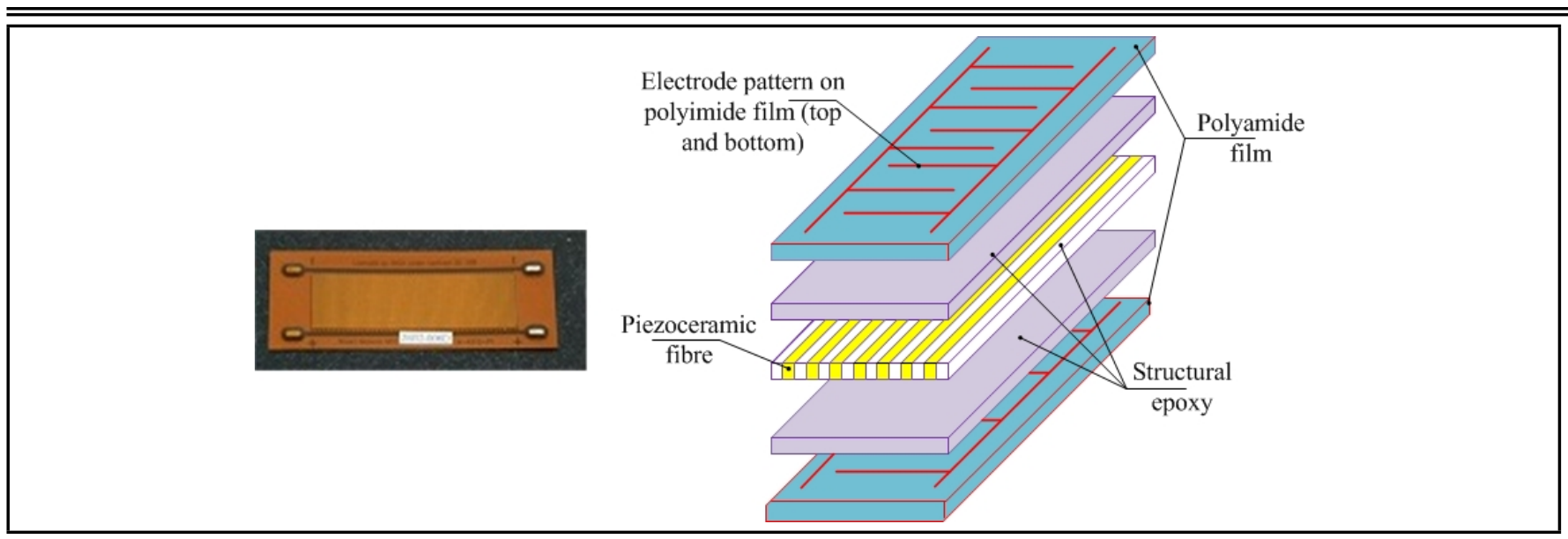

Figure 1. MFC actuator (M4321-P1).

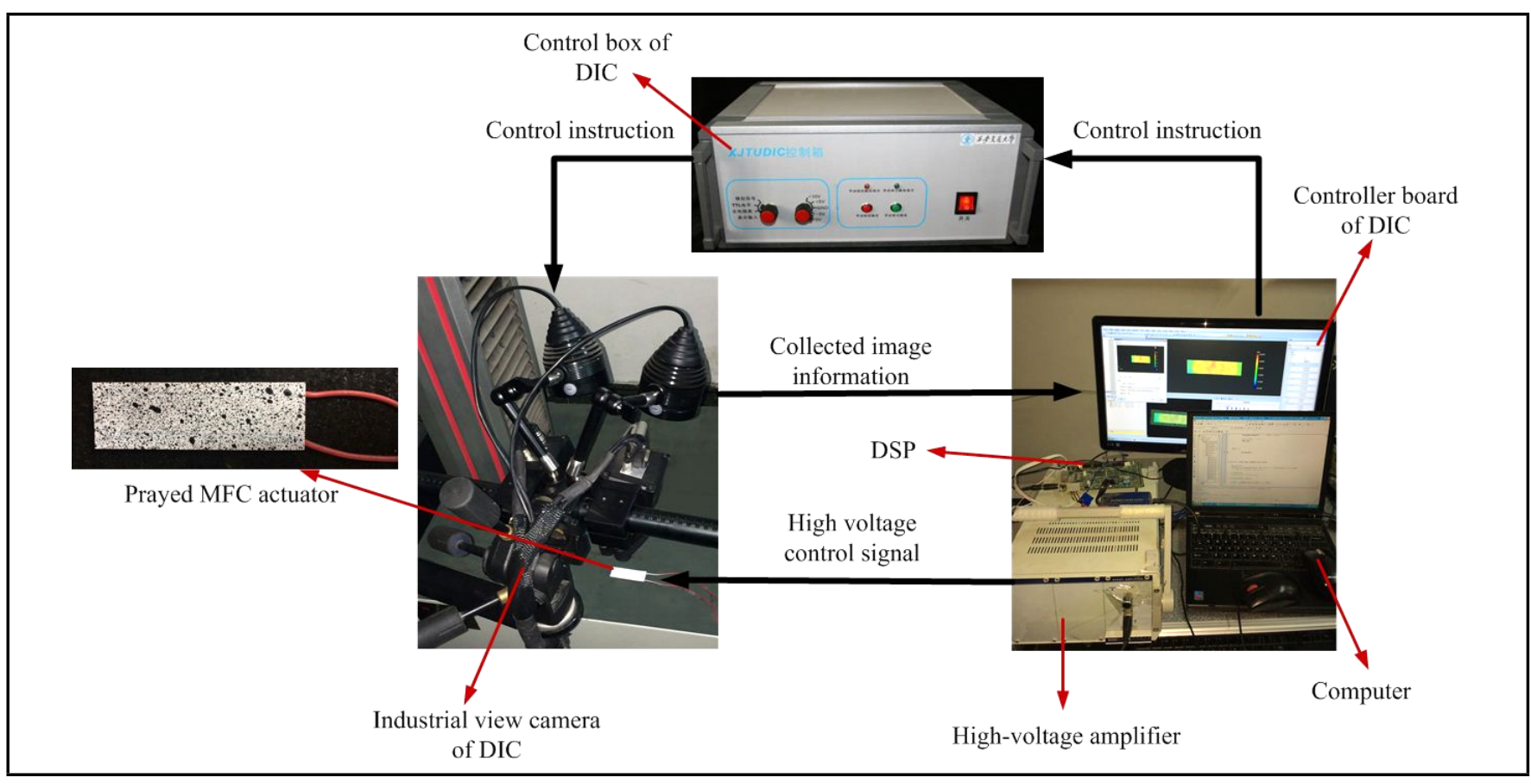

Figure 2. Schematic of the experimental setup.

model of MFC actuator. Williams ${ }^{11}$ provided a detailed nonlinear characterization of the mechanical and piezoelectric behaviour of the MFC actuator. In Bilgen and Friswell's study, ${ }^{26}$ the classical Preisach model was used to predict hysteresis observed in several MFC actuated piezocomposite bimorph devices. In their research, the average prediction error was about $9.3 \%$ for $1 \mathrm{~Hz}$ input signal. The accuracy of the classical Preisach model is limited by the finite number of the hysteresis operators. In other words, the number of data stored determines the accuracy of the model. In the view of this, some modifications should be made to apply classical Preisach model to get better accuracy.

In this paper, the experimental and theoretical study on the electromechanical hysteresis property of MFC actuator is carried out. The paper is organized as follow: in Section 2, the relationships of applied voltage and relative strain under different conditions are obtained experimentally, and the results are used to comprehend the amplitude and frequency to determine how to influence the behaviour of MFC actuator. The hysteresis properties with nonlocal memory including wipingout property and congruency property are also checked based on experimental study. In Section 3, in order to get more accurate prediction of the electromechanical hysteresis behaviour of MFC actuator, the experimental database considering the dynamic factor and the congruency property is used to modify the classical Preisach model. In Section 4, to evaluate the effectiveness of the proposed model, some experiments with different input condition are undertaken. In the experiment, a comparison between the accuracy of the proposed model and that of the classical Preisach model is made; also the influence of the frequency and amplitude on the accuracy of the modified Preisach model is analysed. Finally, conclusions are drawn in Section 5.

\section{EXPERIMENTAL STUDY ON THE ELECTROMECHANICAL HYSTERETIC PROPERTY OF MFC ACTUATOR}

In order to investigate the electromechanical behaviour and hysteresis property with nonlocal memory of MFC actuator, the experimental study is carried out in this section. 


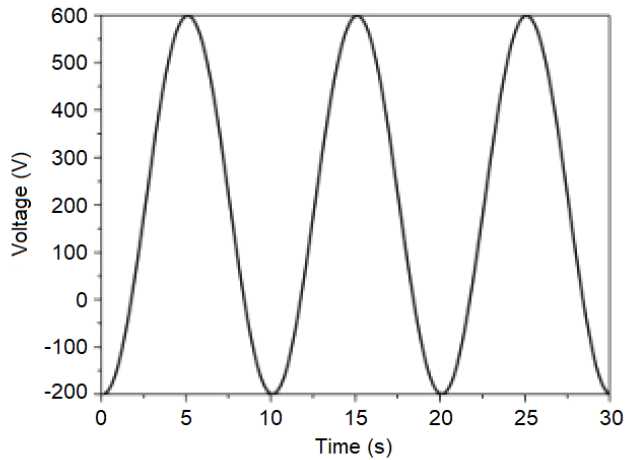

(a) Input voltage vs. time

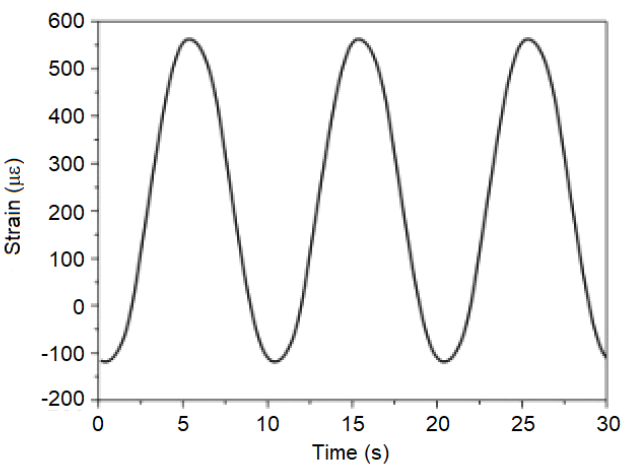

(c) Output strain vs. time

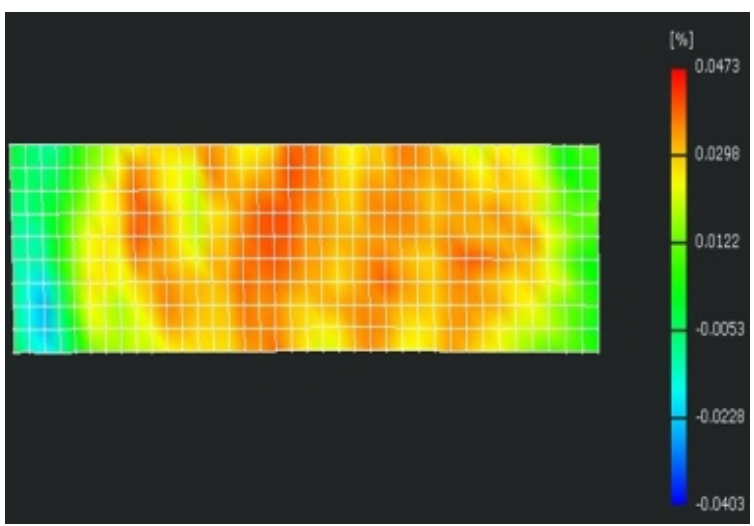

(b) Output strain field

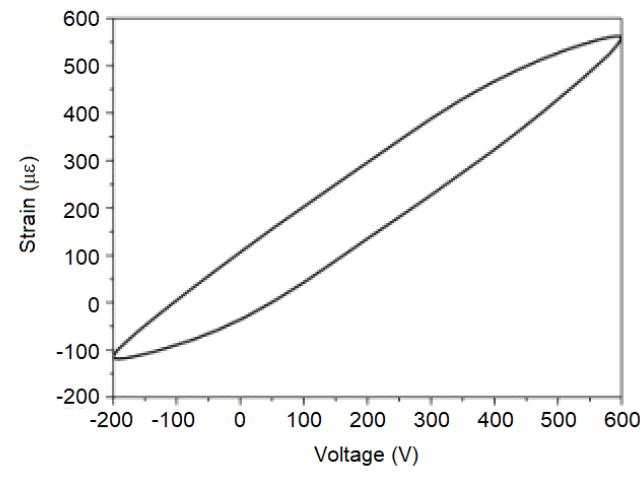

(d) Output strain vs. voltage

Figure 3. Typical test data in the case of Vpp $800 \mathrm{~V}, 200 \mathrm{~V}$ offset and $0.1 \mathrm{~Hz}$ frequency.

Table 1. Experimental scheme at different frequencies $(0.1 \mathrm{~Hz}, 0.5 \mathrm{~Hz}, 1 \mathrm{~Hz}$, $2 \mathrm{~Hz})$.

\begin{tabular}{||c|c|c|c|c|c|c||}
\hline Offset Voltage (V) & 0 & 50 & 100 & 150 & 200 & 250 \\
\hline & 200 & 200 & 200 & 200 & 200 & 200 \\
& 300 & 300 & 300 & 300 & 300 & 300 \\
& 400 & 400 & 400 & 400 & 400 & 400 \\
& 500 & 500 & 500 & 500 & 500 & 500 \\
& 600 & 600 & 600 & 600 & 600 & 600 \\
Peak to Peak & 700 & 700 & 700 & 700 & 700 & 700 \\
Voltage (V) & 800 & 800 & 800 & 800 & 800 & 800 \\
& & 900 & 900 & 900 & 900 & 900 \\
& & & 1000 & 1000 & 1000 & 1000 \\
& & & & 1100 & 1100 & 1100 \\
& & & & & 1200 & 1200 \\
& & & & & & 1300 \\
\hline
\end{tabular}

\subsection{Experiment Setup}

In this experiment, a MFC actuator, a digital signal processing (DSP) system, a high-voltage amplifier and a digital image correlation (DIC) system are used to obtain the relationship of the voltage and strain. The MFC actuator studied in this research is model M4321-P1 produced by the Smart Material Corporation. As shown in Fig. 1, the MFC consists of piezoceramic rods sandwiched between layers of adhesive, electrodes, and polyimide film, and the electrodes are attached to the film in an interdigitated pattern that transfers the applied voltage directly to and from the ribbon shaped rods.

In the experiment, the control signal can be compiled on a controller board in the computer, and then the DSP switches the digital signal to analogue signal and transmits it to the highvoltage amplifier, which is used to amplify the control power to actuate the MFC actuator. The DIC system adopts the non- contact method that uses the three-dimension optical digital speckle technology to acquire the strain of the MFC actuator, and the speckles are prayed on the MFC actuator for optical speckle measurement. The DIC system is composed of two industrial view cameras, a control box, and a computer with calculation software. The detail operational process is depicted as Fig. 2. The DSP receives computer digital signal and transmits it to the high-voltage amplifier to drive the MFC actuator. At the same time, the computer of the DIC system instructs the industrial view cameras through the control box, then the industrial view cameras identify image data and transmit collected information back to the computer, which can analyse the dynamic strain signal to attain strain field.

The experimental results in the case of the harmonic peakto-peak voltage (Vpp) $800 \mathrm{~V}$ combined with $200 \mathrm{~V}$ offset voltage under $0.1 \mathrm{~Hz}$ frequency are shown in Fig. 3. Figure 3(a) represents the harmonic voltage input, Fig. 3(b) shows the stain field obtained directly from the DIC system at one time point, and Fig. 3(c) represents the relative strain response at the midpoint of the MFC actuator. In order to deal with the stable data, only the second cycle of data is used to establish the relationship between input voltage and output strain as shown in Fig. 3(d).

Based on this experiment system, two categories of experiments are carried out. The first one is to obtain the electromechanical behaviours of the MFC actuator, which are measured by actuating the specimen under various voltage amplitudes and frequencies. The other one is to observe and verify the hysteresis property with nonlocal memory of MFC actuator. 


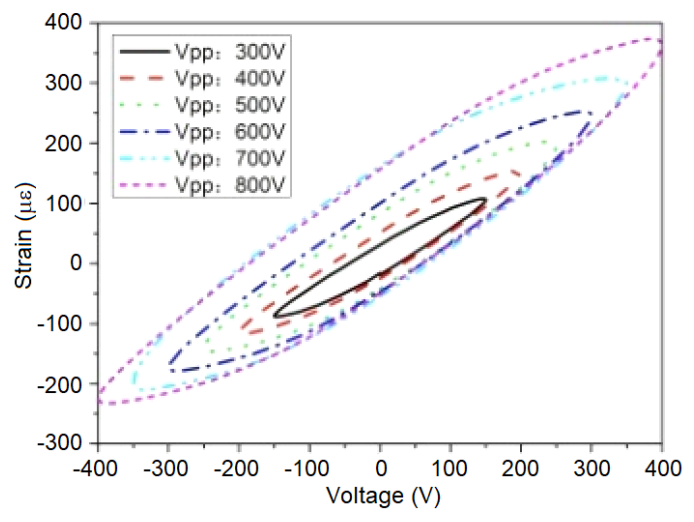

(a) In the case of $0.1 \mathrm{~Hz}$ frequency

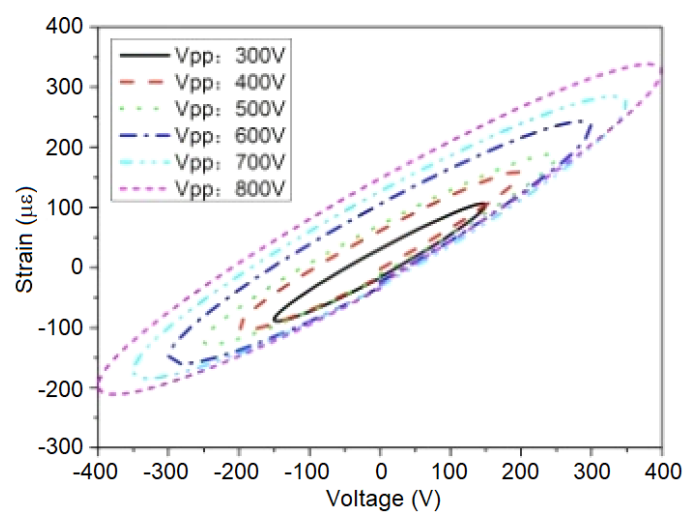

(b) In the case of $1 \mathrm{~Hz}$ frequency

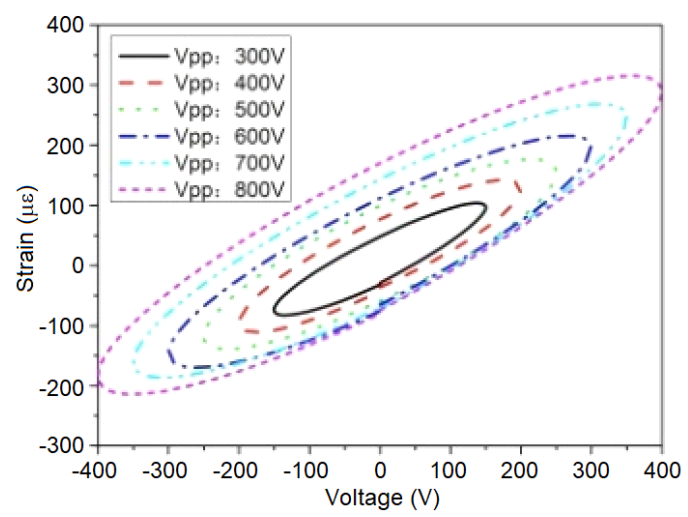

(c) In the case of $2 \mathrm{~Hz}$ frequency

Figure 4. Strain-voltage relationships with different Vpps separately under various frequencies.

\subsection{Electromechanical Behaviour of MFC Actuator}

In order to comprehend the amplitude and frequency to influence the MFC actuator's behaviour, this experiment focuses on investigating electromechanical behaviours under harmonic input voltages with various amplitudes and frequencies. The detail loading texts about harmonic Vpp inputs, offset voltages, and input frequencies $(0.1 \mathrm{~Hz}, 0.5 \mathrm{~Hz}, 1 \mathrm{~Hz}, 2 \mathrm{~Hz})$ are all presented in Table 1.

The experimental results are plotted as shown in Fig. 4 and

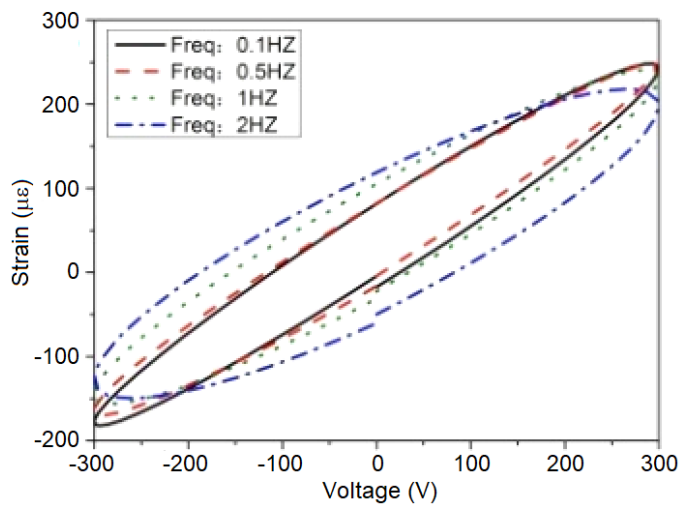

(a) In the case of Vpp $600 \mathrm{~V}$

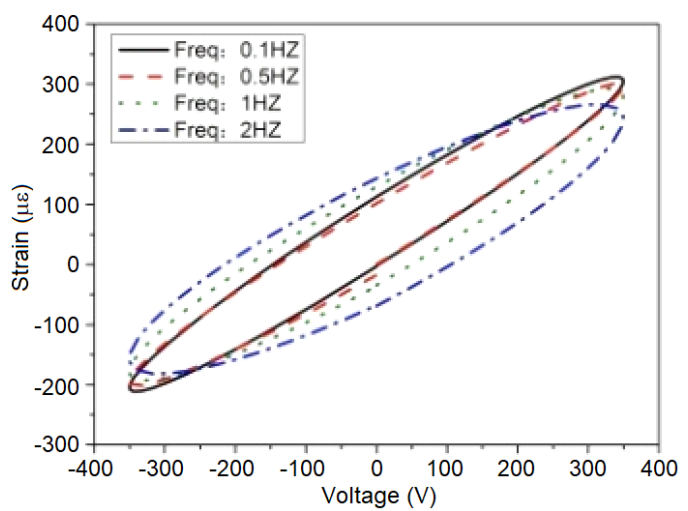

(b) In the case of $\mathrm{Vpp} 700 \mathrm{~V}$

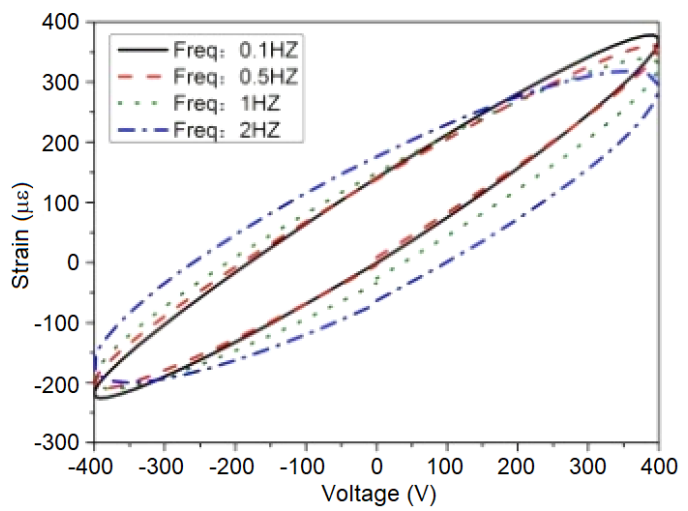

(c) In the case of Vpp $800 \mathrm{~V}$

Figure 5. Strain-voltage relationships with different frequencies separately under various Vpps.

Fig. 5. As shown in Fig. 4, the electromechanical behaviours of the MFC actuator are attained under a constant frequency and increasing harmonic voltages in the range Vpp 300-800 V. Furthermore, hysteresis curves of the MFC actuator under a fixed amplitude and increasing frequencies in the range 0.1$2 \mathrm{~Hz}$ are illustrated in the Fig. 5.

It can be seen from the Fig. 4 that the relationships between voltage and strain exhibit various hysteresis curves under different amplitudes. When the Vpp is increased, the hysteresis curves become bigger, and the characteristics of hysteresis are more obvious, which lead to more internal energy losses. From 


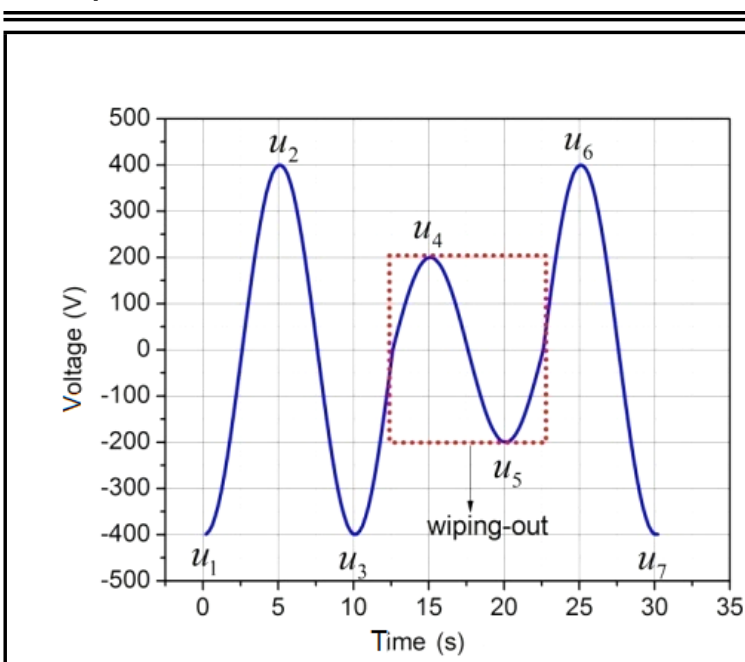

(a) Input voltage vs. time

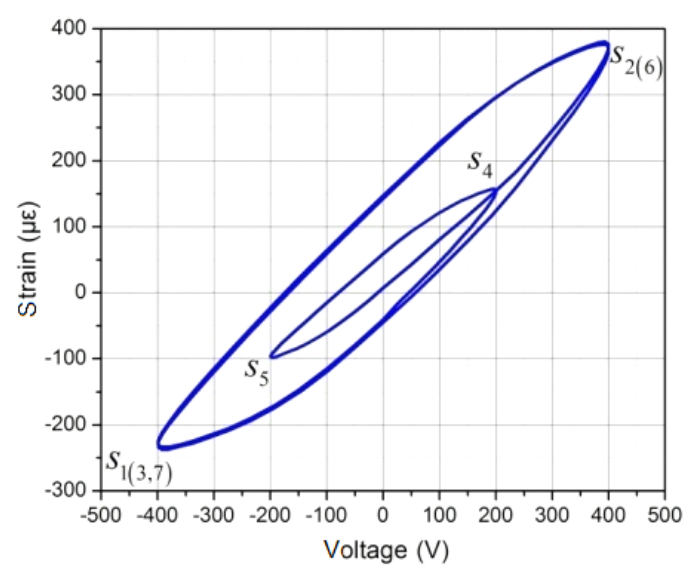

(b) Output strain vs. input voltage

Figure 6. Experiment for wiping-out property of the MFC.

macroscopic viewpoint, the hysteresis loop is a consequence of the effect of domains switching due to the action of the external applied cyclic electric field. Since each domain is a composition of several parallel dipoles, the effective number of dipoles aligned in the direction of the electric field will be altered as domains switch under the action of an external electric field. This domain switching does not occur instantaneously, and it is this delay response that gives rise to the hysteresis loop. Based on the above theory, the higher electric field enhances the domain wall movement, resulting in larger strain.

Figure 5 indicates that the hysteresis effects of MFC actuator are more obvious as the frequencies increase. Intuitively, the length of the minor radius becomes larger with increasing frequencies of the input, while the peak-to-peak output amplitude decreases. In addition, the hysteresis loops turn clockwise with increasing input frequencies. From physical understanding of this phenomenon, the increasing frequencies hasten the accelerated speed of dipoles; therefore, when the domains switch under the action of an external electric field, more internal dipoles delay the response.

Consequently, the hysteresis loops are ellipse-like, and hysteresis characteristics of the MFC actuator are rate-dependent and highly dependent on the amplitudes and frequencies of input voltage.

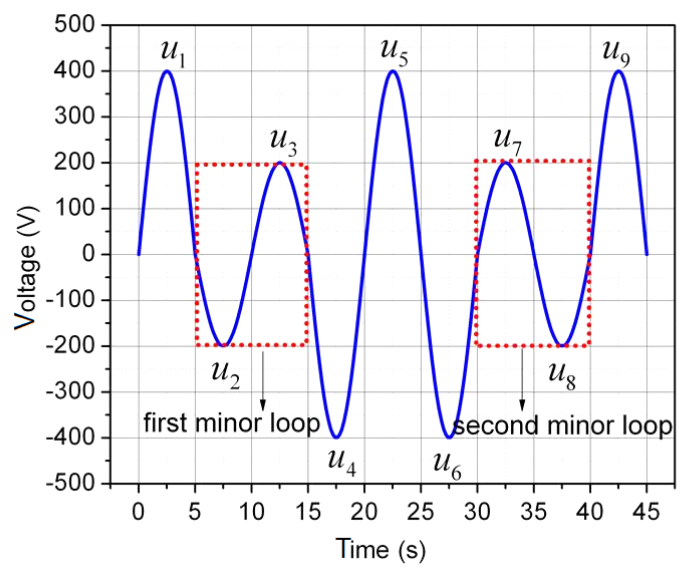

(a) Input voltage vs. time

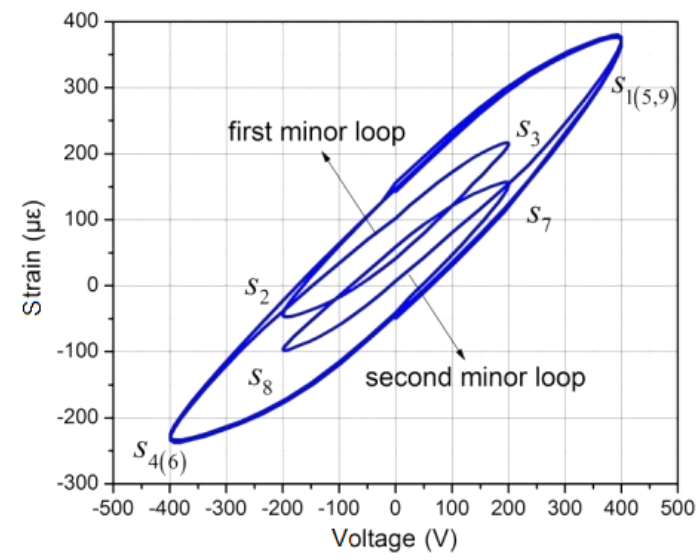

(b) Output strain vs. input voltage

Figure 7. Experiment for congruency property of the MFC.

\subsection{Hysteresis Properties of the MFC Actuator}

Hysteresis with nonlocal memory is a set-valued phenomenon that occurs in piezoelectric materials. Here, the response depends on both the previous extremums as well as the present value of input voltage. The response of the hysteresis phenomenon with nonlocal memory follows some rules that are called wiping-out property and congruency property. In this paper, a series of tests are carried out to verify these properties in MFC materials.

When the piezoelectric material is subjected to a present input extremum, the effects of all previous lesser extremums do not have any impact on future output, and it is called wipingout property. In order to get a better insight into this property, consider the response of the MFC actuators corresponding to the input as shown in Fig. 6(a), and the response of the MFC actuator versus input voltage is shown in Fig. 6(b). In Fig. 6, the points $u_{1}$ to $u_{7}$ and $s_{1}$ to $s_{7}$ correspond to extremums of the input voltage and the output strain, respectively. From Fig. 6(b), the segment between points $s_{2}$ and $s_{3}$ is same as the segment between points $s_{6}$ and $s_{7}$. As expected, the extremums $s_{4}$ and $s_{5}$ are wiped out from the memory of the influencing factors to the later response. In other words, application of such lower peak-to-peak cycles does not affect future 
performance of the MFC actuator.

The congruency property requires that the shapes and inclinations of hysteresis curves formed by the same variations between consecutive local maximum and minimum values are same. To verify this property, the input voltage as shown in Fig. 7(a) is applied to the MFC actuator, and the response is obtained as shown in Fig. 7(b). The points $u_{1}$ to $u_{9}$ and $s_{1}$ to $s_{9}$ correspond to extremums of the input voltage and the output strain, respectively. As shown in Fig. 7(a), $u_{2}$ and $u_{8}$ are equivalence, and $u_{3}$ and $u_{7}$ are also equivalence. Consequently, the first loop and the second loop have the same consecutive maximum and minimum values of input voltage. Figure 7(b) indicates that the congruency property is satisfied because the two minor loops have the same shape and inclination, but they are separated along the vertical output strain axis. This difference is due to the two types of past input histories. Therefore, a translation of the two minor loops in the strain axis will cause the two curves to coincide. Owing to the congruency property, any hysteresis curves that have the same consecutive maximum and minimum values of input voltage are congruent.

In summary, the wiping-out property and the congruency property are two important hysteresis properties that can be observed and verified from the experimental study on the hysteresis property of MFC actuator. It is remarked that these two properties do not only exist in MFC actuator but also in other classes of smart materials.

Consequently, the electromechanical hysteresis behaviour of MFC actuator is complicated, which must be accurately simulated by one appropriate electromechanical model.

\section{MODIFIED PREISACH MODEL OF MFC ACTUATOR}

On the basis of the experimental investigation of hysteresis characteristic in MFC actuator, it is crucial to establish a hysteresis model to describe its electromechanical behaviour accurately. From the experimental results mentioned above, the wiping out property and congruency property are satisfied to meet the classical Preisach model requirement. The classical Preisach model is popular to deal with such electromechanical hysteresis behaviour of piezoelectric material, while the accuracy of the model is limited by the finite number of the hysteresis operators. Due to the limitation of experimental conditions, the number of obtained hysteresis operators could be insufficient to get accurate model hysteresis. Therefore, some modifications are carried out to improve accuracy of the classical Preisach model.

\subsection{Modified Preisach Model}

The mathematical treatment of hysteresis is completely phenomenological with the Preisach model, meaning that it provides no insight to the physical cause of hysteresis, and its mathematical form can be written as

$$
f(t)=\iint_{\alpha \geq \beta} \mu(\alpha, \beta) \gamma_{\alpha \beta}[u(t)] d \alpha d \beta
$$

where $u(t)$ is the input voltage, $f(t)$ is the output strain, and $\gamma_{\alpha \beta}$ is the elemental hysteresis operator with switching values $\alpha$ and $\beta$ whose values are determined by the input voltage $u(t)$.

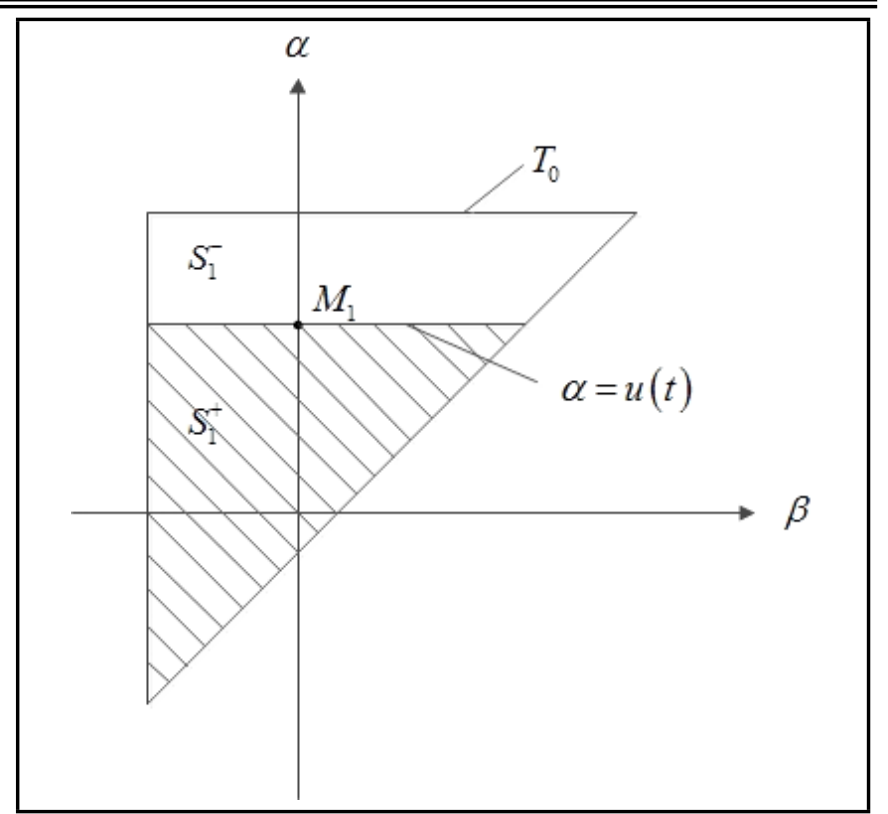

Figure 8. $\alpha$ - $\beta$ half-plane for increasing input signal.

When the input is monotonically increasing from $-\infty$, the output starts at a value of -1 . The output switches at $\alpha$ and stays at +1 until $+\infty$. A similar response results due a monotonically decreasing input, where the output switches from +1 to -1 at the input value of $\beta$. The function $\mu(\alpha, \beta)$ is a weighting function estimated from measured output strain data and is called the Preisach function. The output of the model is, therefore, an integral of $\mu(\alpha, \beta)$ weighted by the hysteresis operators $\gamma_{\alpha \beta}$. The double integration in Eq. (1) is performed such that $u_{\min } \leq \beta \leq \alpha \leq u_{\max }$ where $u_{\min }$ and $u_{\max }$ represent the minimum and maximum value of the input voltage, respectively. The global maximum and minimum of the input voltage and the condition $\alpha \geq \beta$ in Eq. (1) lead to a limiting triangle $T_{0}$ on the $\alpha-\beta$ plane. Within the limiting triangle $T_{0}$, each pair of values $\mu(\alpha, \beta)$ defines a unique operator $\gamma_{\alpha \beta}$ with switching values $\alpha$ and $\beta$.

The Preisach model depends on a special diagram technique that constitutes the mathematical foundation. The graphical method allows the model to detect local input extremums, accumulate them, and choose the right branches of hysteresis nonlinearity. In summary, the geometric definition describes the mechanism of memory formation.

For instance, the input signal starts to increase monotonically to maximum voltage $M_{1}$, which corresponds to the horizontal line $\alpha=u(t)$ moving upward, as shown in Fig. 8. The limiting triangle $T_{0}$ can be divided into two areas, $S_{1}^{+}$and $S_{1}^{-}$. Operators $\gamma_{\alpha \beta}$ that belong to $S_{1}^{+}$are equal to +1 and those that belong to $S_{1}^{-}$are equal to -1 . Therefore, the output strain due to the monotonically increasing input maximum $M_{1}$ represented as $f_{M_{1}}$ can be expressed as

$$
f_{M_{1}}=\iint_{S_{1}^{+}} \mu(\alpha, \beta) d \alpha d \beta-\iint_{S_{1}^{-}} \mu(\alpha, \beta) d \alpha d \beta
$$

Next, the input monotonically decreases from maximum $M_{1}$ to minimum $m_{1}$, corresponding graphically to the vertical line $\beta=u(t)$ moving left through the limiting triangle $T_{0}$ as depicted in Fig. 9. The interface link between $S_{2}^{+}$and $S_{2}^{-}$is called $L(t)$. The hysteresis operators $\gamma_{\alpha \beta}$ that belong to $S_{2}^{+}$ 


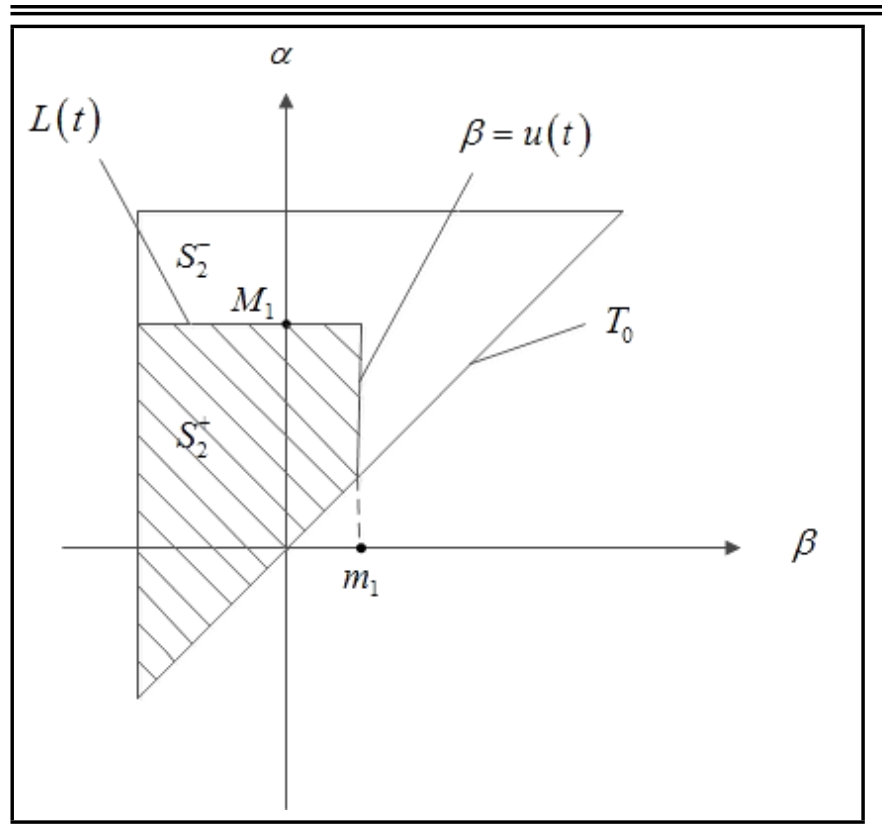

Figure 9. $\alpha$ - $\beta$ half-plane for decreasing input signal.

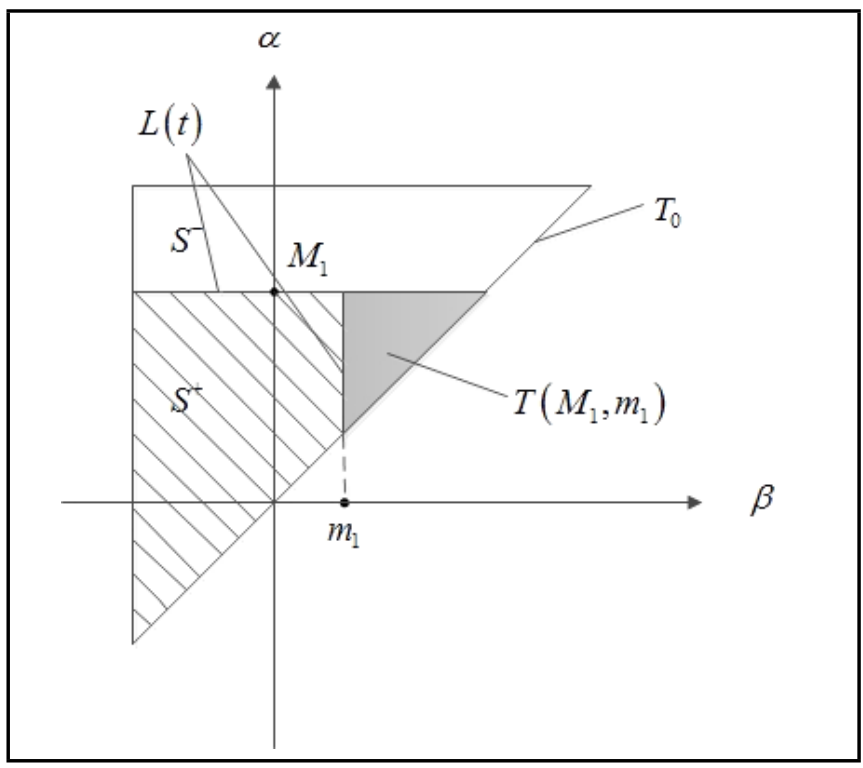

Figure 10. $\alpha-\beta$ half-plane showing the $T\left(M_{1}, m_{1}\right)$.

are equal to +1 and those that belong to $S_{2}^{-}$are equal to -1 . Then the output strain due to the monotonically decreasing input voltage $m_{1}$ is represented as

$$
f_{M_{1} m_{1}}=\iint_{S_{2}^{+}} \mu(\alpha, \beta) d \alpha d \beta-\iint_{S_{2}^{-}} \mu(\alpha, \beta) d \alpha d \beta .
$$

As show in Fig. 10, the monotonic decrease of the input results in the addition of $T\left(M_{1}, m_{1}\right)$ to the set $S^{-}$and subtracts the same area from the set $S^{+}$. So the difference between $f_{M_{1}}$ and $f_{M_{1} m_{1}}$ is denoted as

$$
f_{M_{1}}-f_{M_{1} m_{1}}=2 \iint_{T\left(M_{1}, m_{1}\right)} \mu(\alpha, \beta) d \alpha d \beta
$$

This procedure of updating the interface link $L(t)$ between $S^{+}$and $S^{-}$region within the limiting triangle $T_{0}$ is continued as the input voltage signal varies as shown in Fig. 11. The

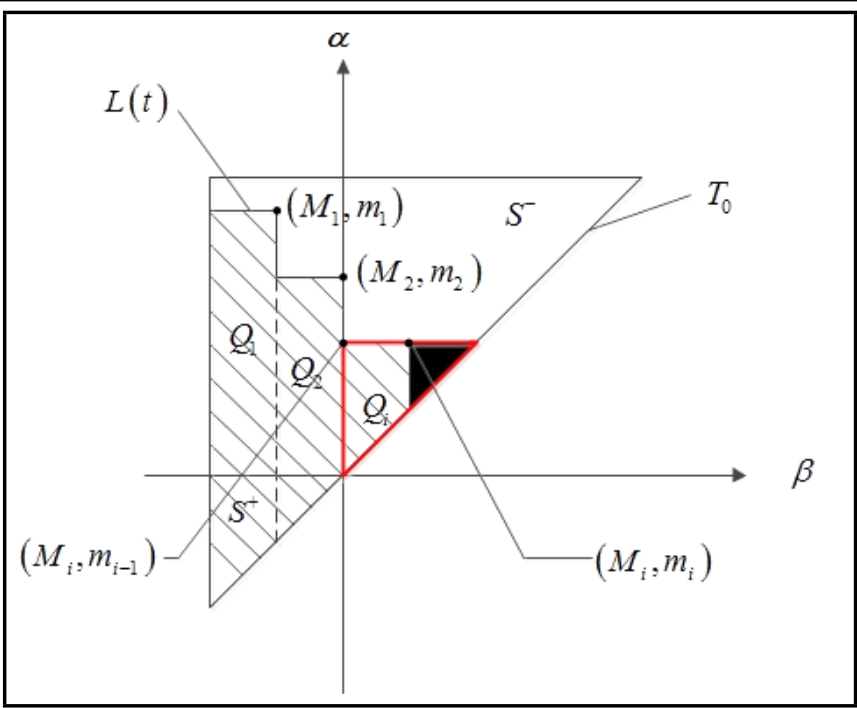

Figure 11. $\alpha$ - $\beta$ half-plane with positive and negative region.

$L(t)$ is a step function whose vertices are denoted $M_{i}$ and $m_{i}$, respectively, which corresponds to the stored input extremums. Here, the wiping out property can be visually quite clearly. For example, if the $M_{2}$ is greater than $M_{1}$, then the horizontal line $\alpha=M_{2}$ would be the highest link of interface link $L(t)$ and the effect of the input $M_{1}$ would clearly be wiped out. Since the hysteresis operator has only two values, 1 and -1 , corresponding to the two regions, $S^{+}$and $S^{-}$, respectively. The classical Preisach model can be written as

$$
f(t)=\iint_{S^{+}} \mu(\alpha, \beta) d \alpha d \beta-\iint_{S^{-}} \mu(\alpha, \beta) d \alpha d \beta .
$$

By adding and subtracting the double integral of the weighting function over the region $S^{+}$, Eq. (5) becomes

$$
f(t)=-\iint_{T_{0}} \mu(\alpha, \beta) d \alpha d \beta+2 \iint_{S^{+}} \mu(\alpha, \beta) d \alpha d \beta ;
$$

where the negative limiting triangle $T_{0}$ is the minimum saturation of output, and the positive region $S^{+}$is sectioned into $n$ trapezoids, referred to as $Q_{i}$, as shown in Fig. 11. As a result, the double integral on the positive region $S^{+}$can be replaced by the sum of these trapezoids, which can change in shape or number with time. Thus, the double integral over $S^{+}$is rewritten as

$$
\iint_{S^{+}} \mu(\alpha, \beta) d \alpha d \beta=\sum_{i=1}^{n} \iint_{T_{0}} \mu(\alpha, \beta) d \alpha d \beta .
$$

Figure 11 shows that each trapezoid $Q_{i}$ is equal to the difference of two triangles, $T\left(M_{i}, m_{i-1}\right)$ and $T\left(M_{i}, m_{i}\right)$. Therefore, the integral over each trapezoid can be expressed as

$$
\iint_{Q_{i}} \mu(\alpha, \beta) d \alpha d \beta=\iint_{T\left(M_{i}, m_{i-1}\right)} \mu(\alpha, \beta) d \alpha d \beta-\iint_{T\left(M_{i}, m_{i}\right)} \mu(\alpha, \beta) d \alpha d \beta .
$$

In Eq. (8), each of these triangles that defines the trapezoids can be expressed by utilizing Eq. (4) as

$$
\iint \mu(\alpha, \beta) d \alpha d \beta=\frac{1}{2}\left(F_{M_{i}}-f_{M_{i} m_{i-1}}\right) ;
$$




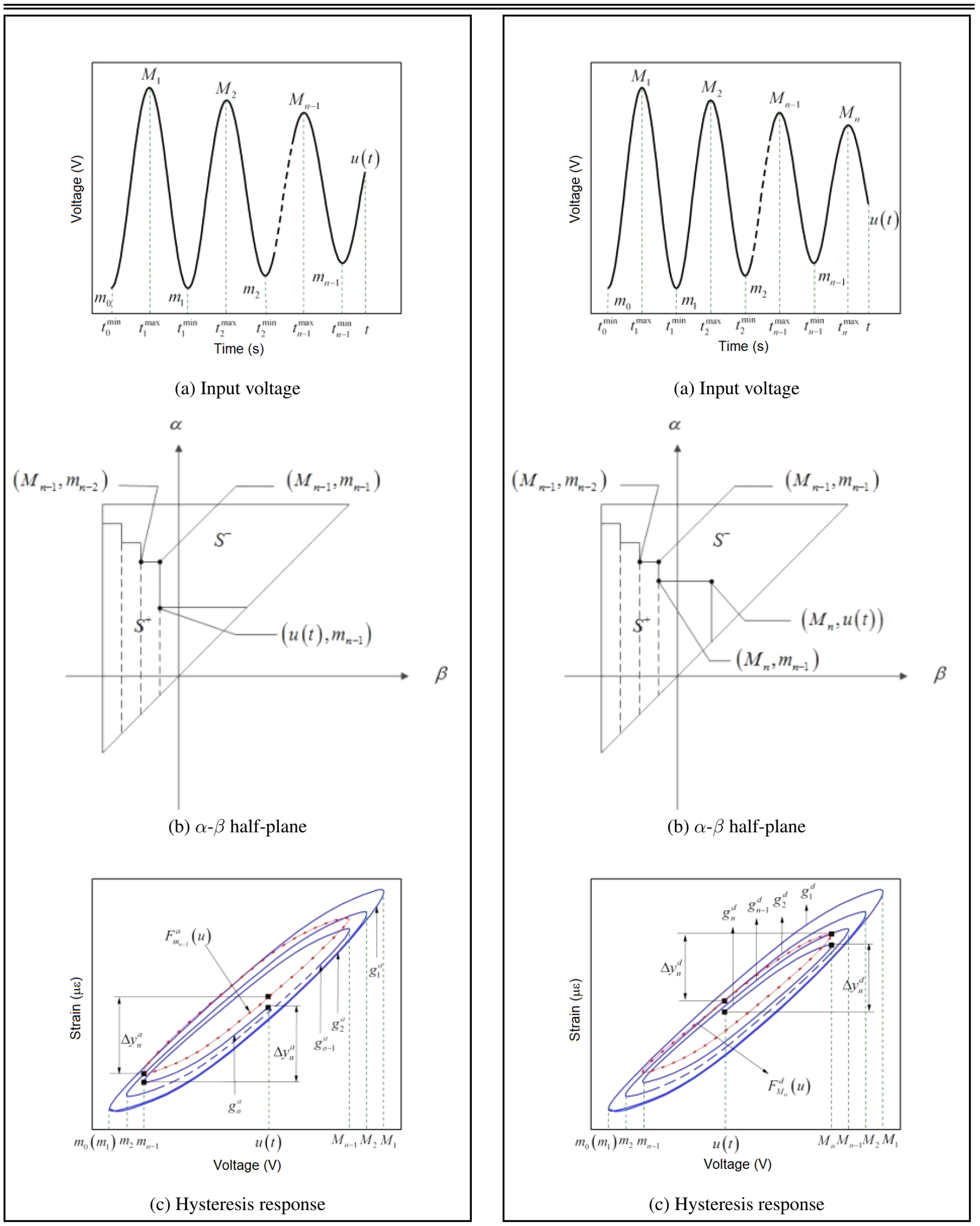

Figure 12. Illustration of the modified Preisach model in the case of increasing segment.

Figure 13. Illustration of the modified Preisach model in the case of decreasing segment.

Then Eq. (8) can be expressed as following

$$
\iint \mu(\alpha, \beta) d \alpha d \beta=\frac{1}{2}\left(F_{M_{i}}-f_{M_{i} m_{i}}\right) .
$$

$$
\iint \mu(\alpha, \beta) d \alpha d \beta=\frac{1}{2}\left(F_{M_{i} m_{i}}-f_{M_{i} m_{i-1}}\right) .
$$


Based on the equations mentioned above, the hysteresis model is established with two cases of state of the input voltage: monotonic increasing input and monotonic decreasing input. First, as shown in Fig. 12(a), consider a harmonic voltage input with gradually decreasing amplitude where the voltage experiences an increasing phase at the current time $t$. Figure 12(b) demonstrates the $\alpha-\beta$ diagram for this input when the final link of $L(t)$ is a horizontal line. Figure 12(c) shows the hysteretic curve of the output strain versus the input voltage, in which $g_{1}^{a}$ is the first ascending segment and $g_{2}^{a}, g_{n-1}^{a}$, and $g_{n}^{a}$ represent the second, $(n-1)$-th, $n$-th ones, respectively. Using Eq. (6), the response to a monotonically increasing input can be written as

$$
\begin{aligned}
f(t)= & -\iint_{T_{0}} \mu(\alpha, \beta) d \alpha d \beta+2 \iint_{Q_{i}} \mu(\alpha, \beta) d \alpha d \beta+ \\
& 2 \iint_{T\left(u(t), m_{n-1}\right)} \mu(\alpha, \beta) d \alpha d \beta ;
\end{aligned}
$$

where the triangle including the current input voltage $u(t)$ is rewritten as

$$
2 \iint_{T\left(u(t), m_{n-1}\right)} \mu(\alpha, \beta) d \alpha d \beta=f_{u(t)}-f_{u(t) m_{n-1}} .
$$

From Eq. (13), the term of $f_{u(t)}-f_{u(t) m_{n-1}}$ is the difference of output strain values at the voltage $u(t)$ and $m_{n-1}$ during the current increasing curve from $m_{n-1}$ to $u(t)$, and the difference is called $\Delta y_{n}^{a}$. Since the classical Preisach model is static and rate-independent in nature, it does not account for the dynamic properties of hysteresis nonlinearity. It is necessary that some modifications should be proposed in order to apply the classical Preisach model to the MFC actuator driven in the quasistatic frequency range. In the view of considering the dynamic factor, the experimental hysteresis curves driving under $0.1 \mathrm{~Hz}$ are used to establish the database to identify the parameter of the model, which will be presented in detail in the following section 3.2. In addition, to predict the hysteretic relationship between the output strain and input voltage by using this experimental database, the congruency property is directly exploited to build the modified Preisach model. According to the congruency property of hysteresis, the difference of $f_{u(t)}$ and $f_{u(t) m_{n-1}}$ in Eq. (13) is congruent with the ascending segment of the hysteresis curve where the maximum and minimum inputs are $M_{n-1}$ and $m_{n-1}$, respectively, as shown in Fig. 12(c). This segment is called $F_{m_{n-1}}^{a}(u(t))$, the value of which is obtained from the previous experiment. Therefore, the difference $\Delta y_{n}^{a}$ can be expressed as follow,

$$
\Delta y_{n}^{a}=F_{m_{n-1}}^{a}(u(t))-F_{m_{n-1}}^{a}\left(m_{n-1}\right) .
$$

Consequently, based on Eq. (11) and Eq. (14), Eq. (12) can be expressed as

$$
\begin{array}{r}
f(t)=f^{-}+\sum_{k=1}^{n-1}\left[f_{M_{k} m_{k}}-f_{M_{k} m_{k-1}}\right]+ \\
F_{m_{n-1}}^{a}(u(t))-F_{m_{n-1}}^{a}\left(m_{n-1}\right)
\end{array}
$$

where the $f^{-}$is the minimum saturation of the output.
Second, as shown in Fig. 13(a), consider a harmonic voltage input with gradually decreasing amplitude where the voltage is during a decreasing phase at the current time $t$. Similarly, Fig. 13(b) demonstrates the $\alpha-\beta$ diagram for this input when the final link of $L(t)$ is a vertical line. The Fig. 13(c) shows the hysteresis curve of the output strain versus the input voltage, in which $g_{1}^{d}, g_{2}^{d}, g_{n-1}^{d}$, and $g_{n}^{d}$ represent the first, second, $(n-1)$ th, and $n$-th descending segment, respectively. Using Eq. (6), the strain response to a monotonically decreasing input can be written as

$$
\begin{array}{r}
f(t)=-\iint_{T_{0}} \mu(\alpha, \beta) d \alpha d \beta+2 \iint_{Q_{i}} \mu(\alpha, \beta) d \alpha d \beta+ \\
2 \iint_{T\left(M_{n}, m_{n-1}\right)} \mu(\alpha, \beta) d \alpha d \beta-2 \iint_{T\left(M_{n}, u(t)\right)} \mu(\alpha, \beta) d \alpha d \beta ;
\end{array}
$$

where the triangle including the current input voltage $u(t)$ is rewritten as

$$
2 \iint_{T\left(M_{n}, u(t)\right)} \mu(\alpha, \beta) d \alpha d \beta=f_{M_{n}}-f_{M_{n} u(t)} .
$$

Here, the term of $f_{M_{n}}-f_{M_{n} u(t)}$ is the difference of output strain values at the voltage $M_{n}$ and $u(t)$ during the current decreasing curve from $M_{n}$ to $u(t)$, and the difference is called $\Delta y_{n}^{d}$. Likewise, based on the congruency property, the difference should be accordant with the descending segment $F_{M_{n}}^{d}(u)$ where the maximum and minimum input are $M_{n}$ and $m_{n-1}$, respectively, as shown in Fig. 13(c). Thus, the difference $\Delta y_{n}^{d}$ can be rewritten as

$$
\Delta y_{n}^{d}=F_{M_{n}}^{d}\left(M_{n}\right)-F_{M_{n}}^{d}(u(t)) \text {. }
$$

Consequently, by using Eq. (9), Eq. (11), and Eq. (18), Eq. (16) can be expressed as

$$
\begin{aligned}
& f(t)=f^{-}+\sum_{k=1}^{n-1}\left[f_{M_{k} m_{k}}-f_{M_{k} m_{k-1}}\right]+ \\
& \quad\left[f_{M_{n}}-f_{M_{n} m_{n-1}}\right]-F_{M_{n}}^{d}\left(M_{n}\right)+F_{M_{n}}^{d}(u(t)) .
\end{aligned}
$$

With the two expressions for hysteresis Eq. (15) and Eq. (19), the values of output strain of the MFC actuator can be obtained based on the information of the strain extremum values and two datasets of the ascending and descending curves.

\subsection{Parameter Identification of the Modified Preisach Model}

In order to employ Eq. (15) and Eq. (19), a set of experimental hysteresis curves of MFC actuator under $0.1 \mathrm{~Hz}$ will be used for the identification, and the databases need to be determined in advance.

Based on the extremum values of measured hysteresis curves, it is found that the dispersing points $f_{M}$ and $f_{M m}$ are akin to the form of three-dimension surface as revealed in Fig. 14. In this sense, the least square method is used to fit these parameters in order to establish the smooth surface function as follows.

$$
f_{M}(M, m)=c_{0}+c_{1} M+c_{2} M^{2}+c_{3} m+c_{4} m^{2}+c_{5} M m
$$

$$
f_{M m}(M, m)=d_{0}+d_{1} M+d_{2} M^{2}+d_{3} m+d_{4} m^{2}+d_{5} M m ;
$$




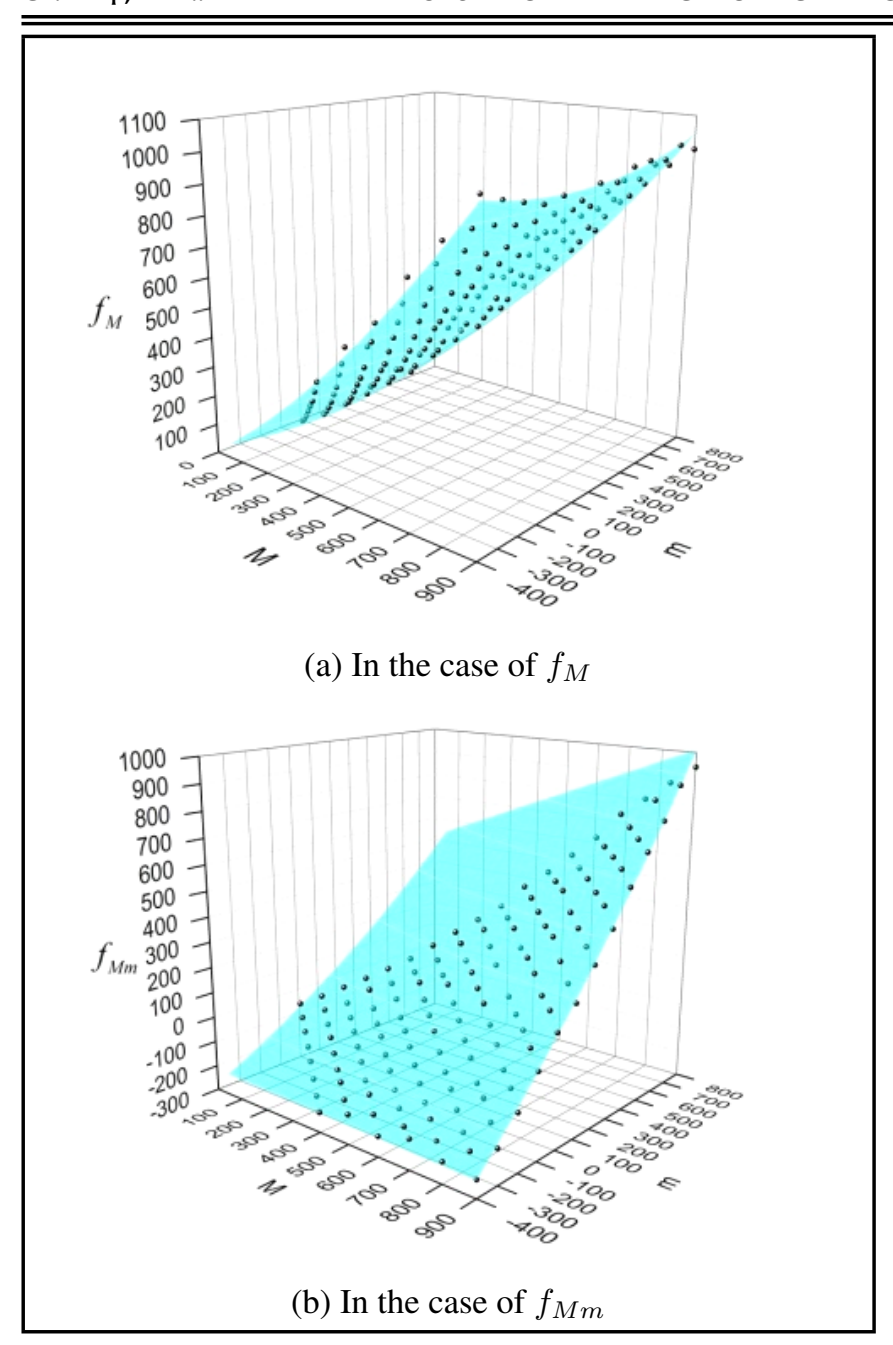

Figure 14. Fitting of the strain-voltage relationship.

where $c_{i}$ and $d_{i}$ are fitting parameters. The unmeasured extremum values $f_{M}$ and $f_{M m}$ can be obtained directly from Eq. (20) and Eq. (21), respectively.

In Eq. (15) and Eq. (19), $F_{m_{n}}^{a}$ and $F_{M_{n}}^{d}$ can be established by polynomial fitting according to the ascending and descending curves of hysteresis curves, respectively. It is given by

$$
\begin{aligned}
& F_{m_{n}}^{a}(u)=\sum_{i=0}^{j} a_{n}^{i} u^{i} ; \\
& F_{M_{n}}^{d}(u)=\sum_{i=0}^{j} d_{n}^{i} u^{i} ;
\end{aligned}
$$

where $m_{n} / M_{n}$ are the initial voltage point of the segments $F_{m_{n}}^{a} / F_{M_{n}}^{d}(u), a_{n}^{i}$ and $d_{n}^{i}$ are the polynomial coefficients of the ascending and descending segment, and $j$ is the polynomial order. Because the hysteresis curves of MFC actuator are smooth, 5-order polynomial functions are enough to describe them accurately. Compared to the method of getting the database in the classical Preisach model, which requires a huge database to get better accuracy, the method of using two polynomial curves for the database is much easier to realize and record. Furthermore, the two polynomial curves that are established by using the hysteresis curves under $0.1 \mathrm{~Hz}$ can take the dynamic factor of experimental data into account.

If the curves $F_{m_{n}}^{a} / F_{M_{n}}^{d}(u)$ in Eq. (15) and Eq. (19) coincide with the ascending and descending segments in the datasets, it

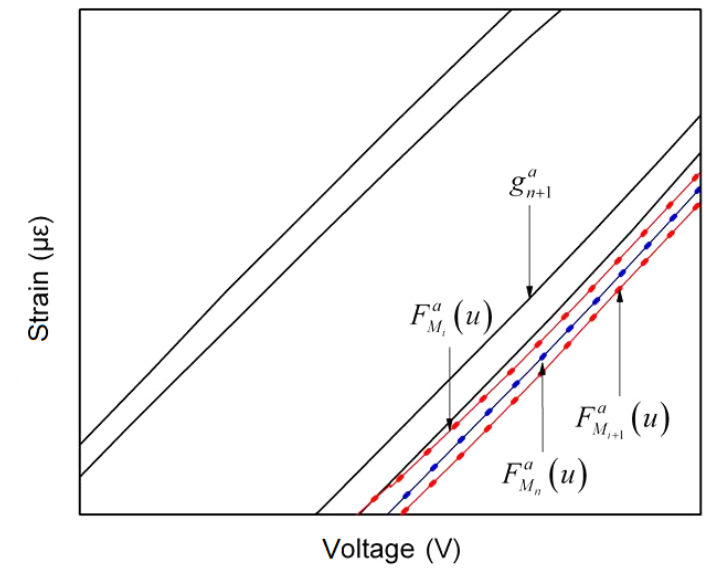

Figure 15. Demonstration of the ascending curve between two consecutive ones.

can be obtained straightforwardly from the available datasets. Otherwise, in the case where initial voltage points $m_{n}$ and $M_{n}$ do not belong to the determined points, the curves $F_{m_{n}}^{a}(u)$ and $F_{M_{n}}^{d}(u)$ will not coincide with any of the ascending and descending segments of the datasets. In this case, an interpolation method is used to obtain these non-determined $F_{m_{n}}^{a}(u)$ and $F_{M_{n}}^{d}(u)$. Assume a specific case that $F_{m_{n}}^{a}(u)$ lies between two consecutive curves $F_{m_{i}}^{a}(u)$ and $F_{m_{i+1}}^{a}(u)$ as demonstrated in Fig. 15. The $F_{m_{n}}^{a}(u)$ is congruent with the ascending segment $g_{n+1}^{a}$. The formulation of the ascending curve $F_{m_{n}}^{a}(u)$ is interpolated based on these curves $F_{m_{i}}^{a}(u)$ and $F_{m_{i+1}}^{a}(u)$ as follow,

$$
F_{m_{n}}^{a}(u)=(1-\alpha) F_{m_{i}}^{a}(u)+\alpha F_{m_{i+1}}^{a}(u)
$$

where $\alpha$ is the scale factor, whose value is defined by

$$
\alpha=\frac{F_{m_{n}}^{a}(u)-F_{m_{i}}^{a}(u)}{F_{m_{i+1}}^{a}(u)-F_{m_{i}}^{a}(u)} .
$$

It is noted that this factor is not constant but varying. In our work, it is proposed to change linearly according to input voltage. Hence, its form can be expressed as

$$
\alpha=\frac{\left(u-m_{n}\right)\left(\left.\alpha\right|_{u=M_{n}}\right)+\left(M_{n}-u\right)\left(\left.\alpha\right|_{u=m_{n}}\right)}{M_{n}-m_{n}} ;
$$

where $\left.\alpha\right|_{u=m_{n}}$ and $\left.\alpha\right|_{u=M_{n}}$ are two boundary values of the scale factor at the inputs $m_{n}$ and $M_{n}$, respectively. At the input $u(t)=m_{m}, f_{M m}\left(M_{n}, m_{n}\right)$ is the value of the ascending segment $F_{m_{n}}^{a}(u)$ at $m_{n}$, which can be obtained from Eq. (21). Therefore, from Eq. (25), the scale factor $\alpha$ at this input value is determined by

$$
\left.\alpha\right|_{u=m_{n}}=\frac{f_{M m}\left(M_{n}, m_{n}\right)-F_{m_{i}}^{a}\left(m_{n}\right)}{F_{m_{i+1}}^{a}\left(m_{n}\right)-F_{m_{i}}^{a}\left(m_{n}\right)} .
$$

Likewise, at the input value $u(t)=M_{m}$, from Eq. (25), the scale factor is obtained by

$$
\left.\alpha\right|_{u=M_{n}}=\frac{f_{M}\left(M_{n}, m_{n}\right)-F_{m_{i}}^{a}\left(M_{n}\right)}{F_{m_{i+1}}^{a}\left(M_{n}\right)-F_{m_{i}}^{a}\left(M_{n}\right)} ;
$$

where $f_{M}\left(M_{n}, m_{n}\right)$ can be obtained from Eq. (20). 
Table 2. Comparison of the RMSEs between the modified Preisach model and the classical Preisach model.

\begin{tabular}{||c|c|c|c|c|c||}
\hline \multirow{2}{*}{} & \multicolumn{5}{|c|}{$\begin{array}{c}\text { RMSE }(\mu \varepsilon) \\
\text { The proposed model / The classical Preisach model }\end{array}$} \\
\cline { 2 - 6 } & $\begin{array}{c}\text { Vpp: 800 V-400 V } \\
\text { Offset: 0 V }\end{array}$ & $\begin{array}{c}\text { Vpp: } 900 \mathrm{~V}-500 \mathrm{~V} \\
\text { Offset: } 50 \mathrm{~V}\end{array}$ & $\begin{array}{c}\text { Vpp: } 1100 \mathrm{~V}-700 \mathrm{~V} \\
\text { Offset: } 150 \mathrm{~V}\end{array}$ & $\begin{array}{c}\text { Vpp: } 1200 \mathrm{~V}-800 \mathrm{~V} \\
\text { Offset: } 200 \mathrm{~V}\end{array}$ & \multicolumn{2}{|c|}{ Average } \\
\hline $0.1 \mathrm{~Hz}$ & $15.91 / 31.56$ & $15.15 / 35.74$ & $12.94 / 55.39$ & $23.79 / 64.61$ & $17.01 / 46.83$ \\
$0.5 \mathrm{~Hz}$ & $13.02 / 32.27$ & $13.91 / 30.59$ & $30.36 / 47.52$ & $38.11 / 56.08$ & $23.92 / 41.62$ \\
$1 \mathrm{~Hz}$ & $20.57 / 29.81$ & $17.49 / 27.81$ & $51.43 / 74.45$ & $49.30 / 69.82$ & $34.69 / 50.47$ \\
$2 \mathrm{~Hz}$ & $49.37 / 53.13$ & $46.63 / 55.52$ & $58.81 / 62.37$ & $87.02 / 88.06$ & $60.45 / 64.77$ \\
Average & $24.71 / 36.69$ & $23.29 / 37.41$ & $38.45 / 59.93$ & $49.62 / 69.64$ & \\
\hline
\end{tabular}

Similarly, the procedure for interpolating the descending curve $F_{M_{n}}^{d}(u)$ lying between two consecutive $F_{M_{i}}^{d}(u)$ and $F_{M_{i+1}}^{d}(u)$ can be conducted as above. The expression is obtained as

$$
F_{M_{n}}^{d}(u)=(1-\beta) F_{M_{i}}^{d}(u)+\beta F_{M_{i+1}}^{d}(u)
$$

where $\beta$ is the scale factor, whose value is proposed to change linearly as follows,

$$
\beta(u)=\frac{\left(u-M_{n}\right)\left(\left.\beta\right|_{u=m_{n-1}}\right)+\left(m_{n-1}-u\right)\left(\left.\beta\right|_{u=M_{n}}\right)}{m_{n-1}-M_{n}} .
$$

Here, $\left.\beta\right|_{u=M_{n}}$ and $\left.\beta\right|_{u=m_{n-1}}$ are, respectively, two boundary values of the scale factor at the inputs $M_{n}$ and $m_{n-1}$ that are determined in the following form,

$$
\begin{aligned}
&\left.\beta\right|_{u=M_{n}}= \frac{f_{M}\left(M_{n}, m_{n-1}\right)-F_{M_{i}}^{d}\left(M_{n}\right)}{F_{M_{i+1}}^{d}\left(M_{n}\right)-F_{M_{i}}^{d}\left(M_{n}\right)} ; \\
&\left.\beta\right|_{u=m_{n-1}}=\frac{f_{M m}\left(M_{n}, m_{n-1}\right)-F_{M_{i}}^{d}\left(m_{n-1}\right)}{F_{M_{i+1}}^{d}\left(m_{n-1}\right)-F_{M_{i}}^{d}\left(m_{n-1}\right)} .
\end{aligned}
$$

where the $f_{M}\left(M_{n}, m_{n-1}\right)$ and $f_{M m}\left(M_{n}, m_{n-1}\right)$ can be obtained from Eq. (20) and Eq. (21), respectively. Consequently, Eq. (22) with the interpolation technique Eq. (24) and Eq. (23) with interpolation scheme Eq. (29) constitute the approach to obtain the ascending segment and descending segment.

In summary, the output strain of the MFC actuator can be obtained through the two expressions Eq. (15) and Eq. (19) in the cases of monotonic increase and monotonic decrease of input voltage, respectively. In addition, in order to utilize the surface function and the curve-fitting functions, the database of hysteresis curves need to be determined in advance.

\section{VERIFICATION AND DISCUSSION OF THE MODIFIED PREISACH MODEL}

In order to evaluate the accuracy of the proposed model, several experiments with different harmonic waveforms of gradually decreasing amplitude are employed. In the experiment, the simulation responses using the proposed model are compared with those using the classical Preisach model, and prediction error analysis is carried out through different applied frequencies and voltage amplitudes. In addition, the influence of the frequency and amplitude on the accuracy of the modified Preisach model is analysed.

Statistics about estimation errors of the modified Preisach model and the classical Preisach model for the experimental results are illustrated in Table 2. The article employs the rootmean-square error (RMSE) herein to be taken as the distance between the simulation and experiment results, which is governed by

$$
\mathrm{RMSE}=\sqrt{\frac{1}{n} \sum_{i=1}^{n}\left(f_{\mathrm{sim}, i}-f_{\exp , i}\right)^{2}}
$$

where $n$ is the number of data points, and $f_{\operatorname{sim}, i} / f_{\exp , i}$ is the strain from simulation/experiment data point that is indexed by the subscript sim, $i$ /exp, $i$.

In Table 2, partial simulation accuracies by the proposed model are compared with the results by the classical Preisach model. The input is set as harmonic voltage with gradually decreasing amplitude in 5 cycles. When the amplitude is from Vpp $800 \mathrm{~V}$ to Vpp $400 \mathrm{~V}$ with $0.1 \mathrm{~Hz}$ frequency and $0 \mathrm{~V}$ offset, the RMSE using the proposed model is $15.91 \mu \varepsilon$. In contrast, the RMSE using the classical model is a larger value by $31.56 \mu \varepsilon$. Thus, the proposed model is superior to the classical one, which is also verified in Fig. 16. In Fig. 16(a), the response from the proposed model agrees very well with the actual response measured from the MFC actuator. However, the simulation result by the classical model is not identical with test result as shown in Fig. 16(b). In addition, the strain rate of the hysteresis curves is shown in Fig. 16(c) and Fig. 16(d). The strain rate derived by the modified Preisach model matches better with experimental result than that derived by the classical model. Intuitively, inclinations of relationships between voltage and strain rate from experiment and modified model are higher than the classical model. The results indicate that the modified Preisach model takes dynamic influence into account. After that, this input voltage is also carried out under $0.5 \mathrm{~Hz}$, $1 \mathrm{~Hz}$ and $2 \mathrm{~Hz}$ frequency respectively, and the results in Table 2 are important to note that the modified Presiach model gives better prediction than the classical one.

Considering the results of the different Vpps and offset voltages under $0.5 \mathrm{~Hz}$ in Table 2, the average RMSE using the proposed method is $23.92 \mu \varepsilon$. The average RMSE using the classical method is $41.62 \mu \varepsilon$. In this sense, the modified model is better than the classical one. Figure 17(a) and Fig. 17(b) illustrate the resultant responses in the case of Vpp $1200 \mathrm{~V}-800 \mathrm{~V}$, $200 \mathrm{~V}$ offset and $0.5 \mathrm{~Hz}$ frequency. It is shown that the simulation response from the modified Preisach model agrees well with the experimental result and the accuracy is better than that of the classical Preisach model. Likewise, the strain rate of these hysteresis curves is shown in Fig. 17(c) and Fig. 17(d). The strain rate derived by the modified Preisach model matches better with experimental data than that derived by the classical one. Thus, we observe that the modified model is superior to the classical model.

In addition, Fig. 18 illustrates the variation tendency of the error over time of the modified Preisach model. Error is set as the difference between the simulation and experiment data. 


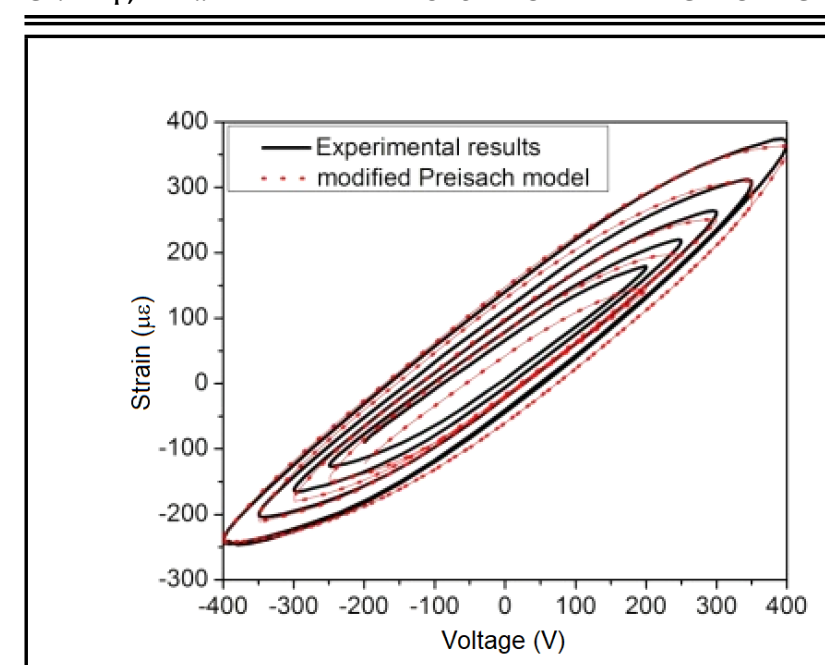

(a) Strain-voltage relationship in the case of the modified Preisach model

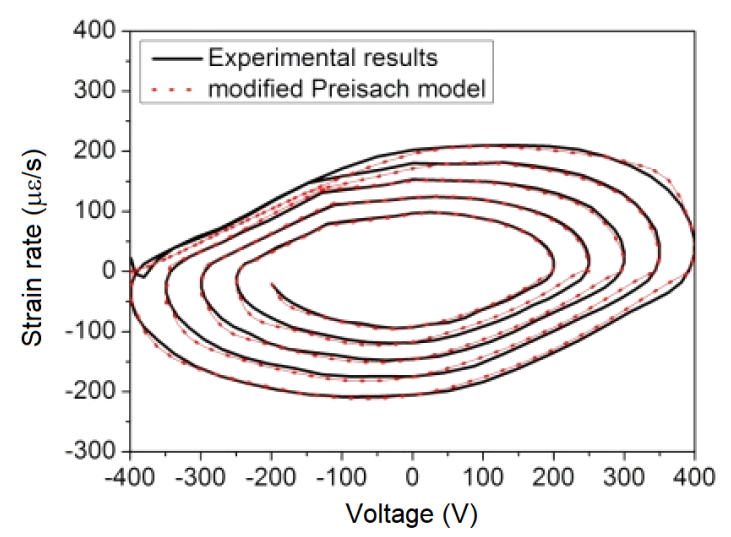

(c) Strain rate-voltage relationship in the case of the modified Preisach model

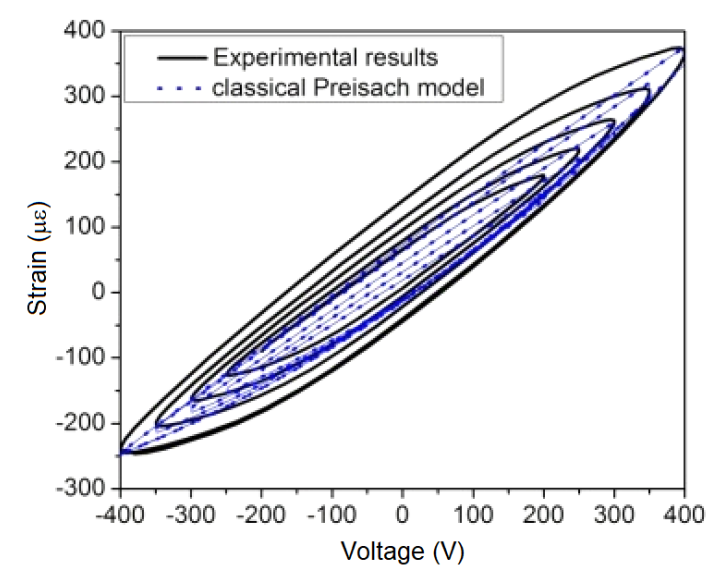

(b) Strain-voltage relationship in the case of the classical Preisach model

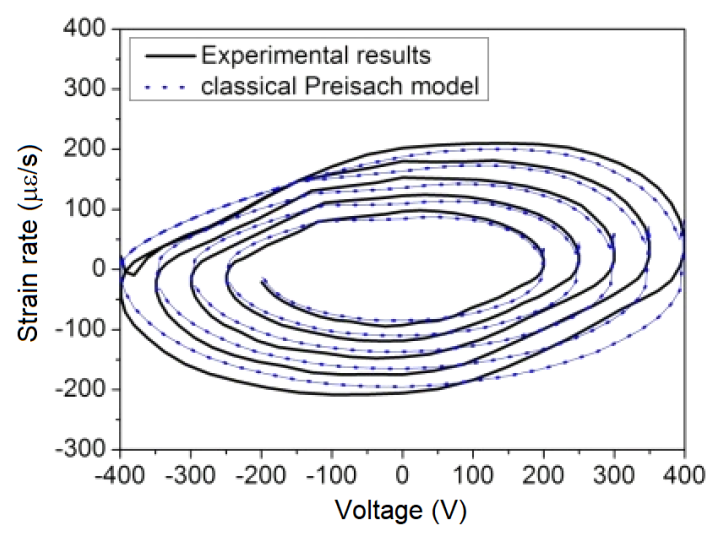

(d) Strain rate-voltage relationship in the case of the classical Preisach model

Figure 16. Experimental results in the case of Vpp $800 \mathrm{~V}-400 \mathrm{~V}$, offset $0 \mathrm{~V}$ and $0.1 \mathrm{~Hz}$.

Figure 18(a) presents error as a function of input frequency and time period, and Fig. 18(b) illustrates error as a function of amplitude and time. These figures indicate that the errors of the modified Preisach model gradually rise with increasing frequency and amplitude.

\section{CONCLUSIONS}

In this paper, the modified Preisach model is proposed to predict the hysteresis of the MFC actuator. First, a series of tests under harmonic voltage input with different frequencies and voltage amplitudes are conducted to investigate the electromechanical behaviour and hysteresis property of the MFC actuator. The experimental results indicate that characteristics of hysteresis curves are sensitive to the varying voltage amplitudes and frequencies. The wiping-out property and the congruency property are also verified to exist in MFC actuator through experimental study. Second, by using the congruency property and the identified database, which considers the dynamic factor, the modified Preisach model to predict the hysteresis behaviour of MFC actuator is proposed. In order to apply the proposed model, the databases of extremums as well as ascending and descending segments are experimentally predetermined. Finally, in order to evaluate the effectiveness of the proposed model, experiments with different schemes of input voltage are carried out. In the test, the simulation responses using the proposed model are compared with those using the classical Preisach model. The proposed Preisach model is in better agreement with experimental data, illustrating the accuracy of the proposed model better than classical Preisach one. It is expected that the MFC actuator associated with the modified Preisach model can be effectively utilized to vibration control system.

\section{ACKNOWLEDGEMENTS}

This research is financially supported by the National Natural Science Foundation, People's Republic of China, grant No. 11172226, this support is gratefully acknowledged.

\section{REFERENCES}

1 Deraemaeker, A., Nasser, H., Benjeddou, A. Mixing rules for the piezoelectric properties of macro fiber composite, Journal of Intelligent Material Systems and Structures, 20 (12), 1475-1482, (2009). https://dx.doi.org/10.1177/1045389X09335615

2 Deraemaeker, A., Nasser, H. Numerical evaluation of the 


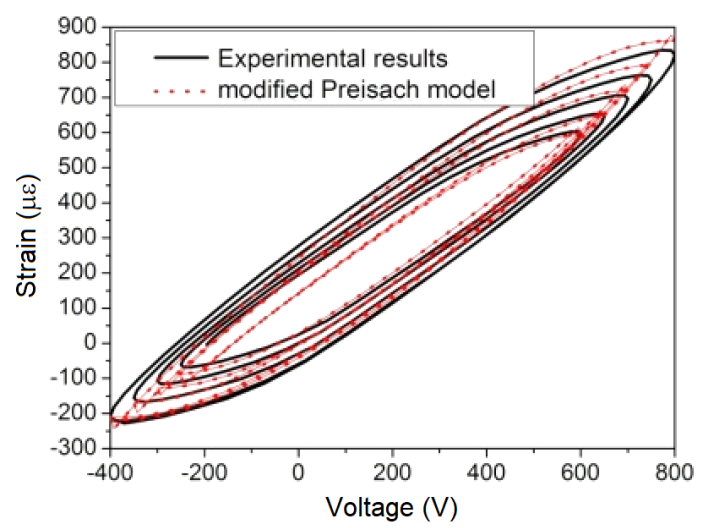

(a) Strain-voltage relationship in the case of the modified Preisach model

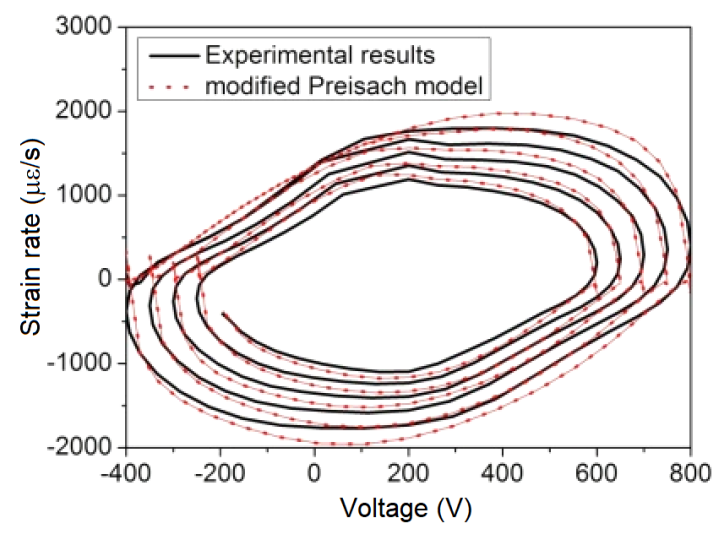

(c) Strain rate-voltage relationship in the case of the modified Preisach model

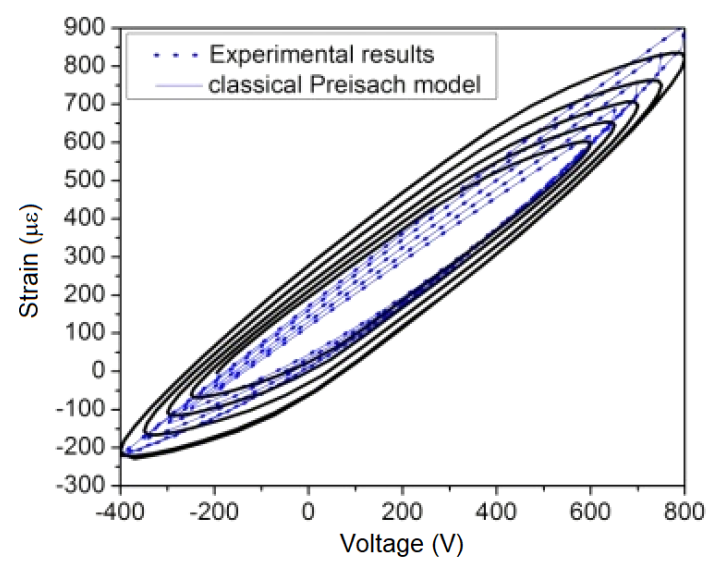

(b) Strain-voltage relationship in the case of the classical Preisach model

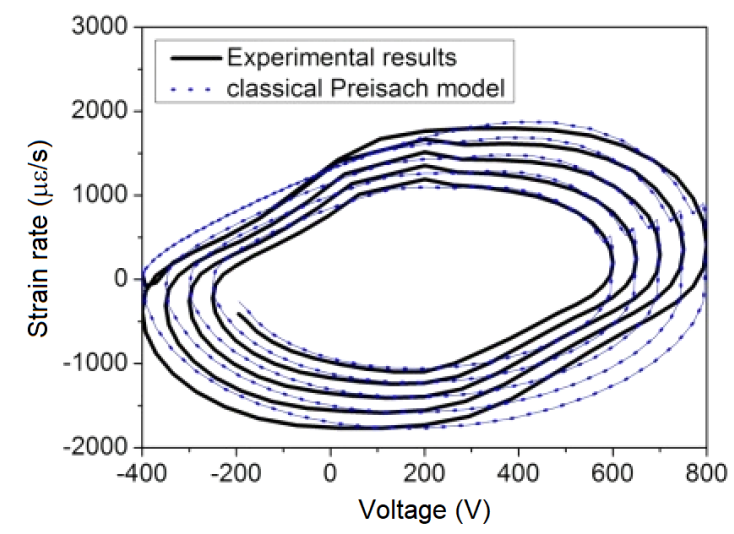

(d) Strain rate-voltage relationship in the case of the classical Preisach model

Figure 17. Experimental results in the case of Vpp $1200 \mathrm{~V}-800 \mathrm{~V}$, offset $200 \mathrm{~V}$ and $0.5 \mathrm{~Hz}$.

equivalent properties of Macro Fiber Composite transducers using periodic homogenization, International Journal of Solids and Structures, 47 (24), 3272-3285, (2010). https://dx.doi.org/10.1016/j.ijsolstr.2010.08.006

3 Bilgen, O., Kochersberger, K. B., Inman, D. J. Macrofiber composite actuated simply supported thin airfoils, Smart Materials and Structures, 19 (5), 055010, (2010). https://dx.doi.org/10.1088/0964-1726/19/5/055010

4 Schrock, J., Meurer, T., Kugi, A. Control of a flexible beam actuated by macro-fiber composite patches: I. Modeling and feedforward trajectory control, Smart Materials and Structures, 20 (1), 015015, (2011). https://dx.doi.org/10.1088/0964-1726/20/1/015015

5 Schrock, J., Meurer, T., Kugi, A. Control of a flexible beam actuated by macro-fiber composite patches: II. Hysteresis and creep compensation, experimental results, Smart Materials and Structures, 20 (1), 015016, (2011). https://dx.doi.org/10.1088/0964-1726/20/1/015016

6 Sohn, J. W., Choi, S. B., Kim, H. S. Vibration control of smart hull structure with optimally placed piezoelectric composite actuators, International Jour- nal of Mechanical Sciences, 53 (8), 647-659, (2011). https://dx.doi.org/10.1016/j.ijmecsci.2011.05.011

7 Cui, L., Liu, Y., Soh, C. K. Macro-fiber composite-based structural health monitoring system for axial cracks in cylindrical structures, Journal of Intelligent Material Systems and Structures, 1045389X13494929, (2013). https://dx.doi.org/10.1177/1045389X13494929

8 Min, J., Park, S., Yun, C. B. Impedance-based structural health monitoring incorporating neural network technique for identification of damage type and severity, Engineering Structures, 39, 210-220, (2012). https://dx.doi.org/10.1016/j.engstruct.2012.01.012

9 Yang, Y., Tang, L., Li, H. Vibration energy harvesting using macro-fiber composites, Smart Materials and Structures, 18 (11), 115025, (2009). https://dx.doi.org/10.1016/j.engstruct.2012.01.012

10 Meehan, A., Gao, H., Lewandowski, Z. Energy harvesting with microbial fuel cell and power management system, IEEE Transactions on Power Electronics, 26 (1), 176-181, (2011). https://dx.doi.org/10.1109/ECCE.2009.5316034 


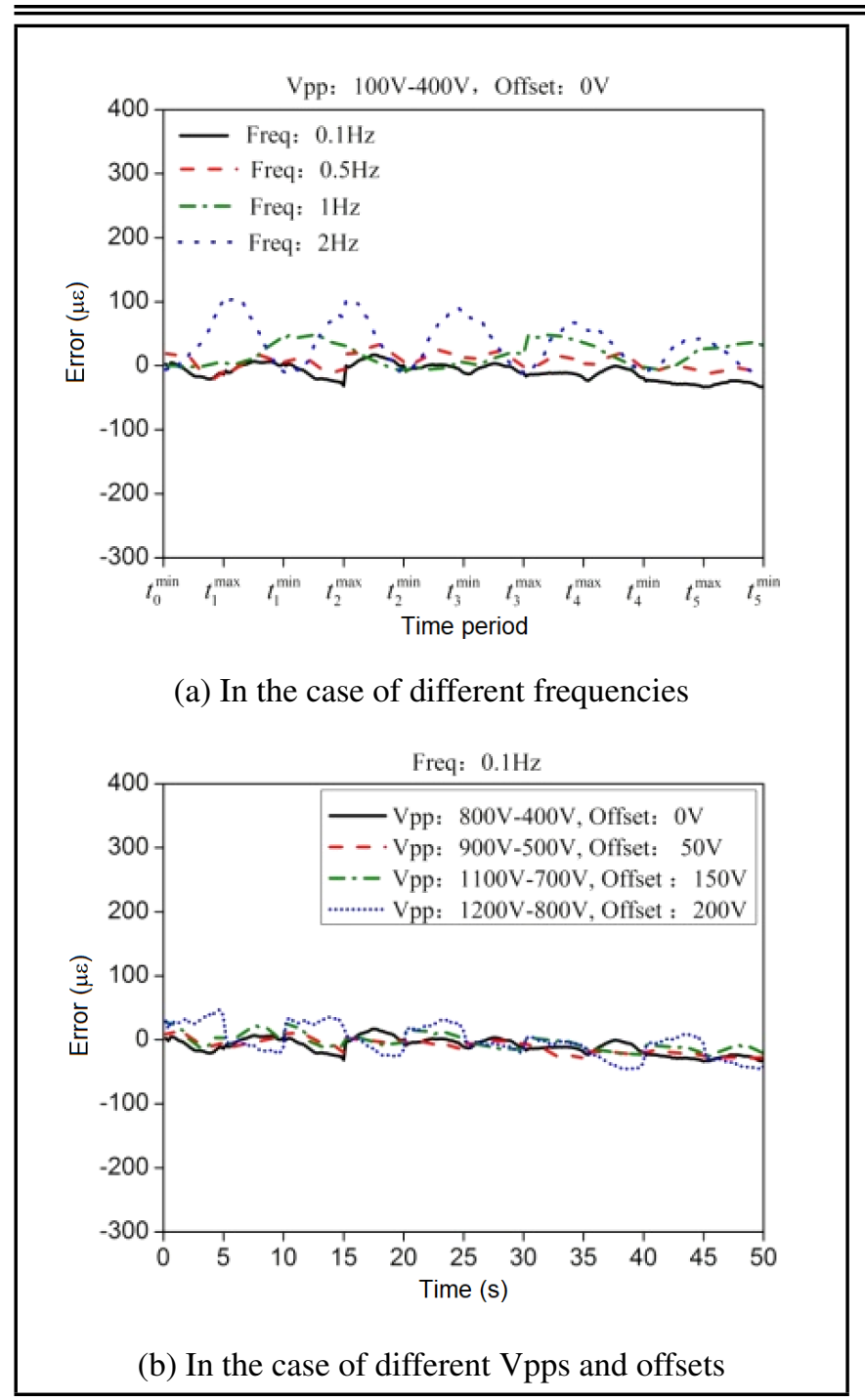

Figure 18. Error analysis.

11 Williams, R. B. Nonlinear mechanical and actuation characterization of piezoceramic fiber composites, Doctoral dissertation, Virginia Polytechnic Institute and State University. Blacksburg, VA, (2004). https://dx.doi.org/10.18297/etd/855

12 Hegewald, T., Kaltenbacher, B., Kaltenbacher, M. Efficient modeling of ferroelectric behavior for the analysis of piezoceramic actuators, Journal of Intelligent Material Systems and Structures, 19 (10), 1117-1129, (2007). https://dx.doi.org/10.1177/1045389X07083608

13 Dupre, L. R., Van Keer, R., Melkebeek Jan AA. Identification of the relation between the material parameters in the Preisach model and in the Jiles-Atherton hysteresis model, Journal of Applied Physics, 85 (8), 4376-4378, (1999). https://dx.doi.org/10.1063/1.369789

14 Smith, R. C., Hatch, A. G., Mukherjee, B. A homogenized energy model for hysteresis in ferroelectric materials: General density formulation, Journal of Intelligent Material Systems and Structures, 16 (9), 713-732, (2005). https://dx.doi.org/10.1177/1045389X05054789
15 Zhang, C., Qiu, J., Chen, Y. Modeling hysteresis and creep behavior of macro fiber composite-based piezoelectric bimorph actuator, Journal of Intelligent Material Systems and Structures, 24 (3), 369-377, (2013). https://dx.doi.org/10.1177/1045389X12460337

16 Goldfarb, M., Celanovic, N. A Lumped Parameter Electromechanical Model for describing the nonlinear hysic ur of piezoelectric actuators, Journal of Dynamic Systems, Measurement, and Control, 119 (3),119-479, (1997). https://dx.doi.org/10.1115/1.2801282

17 Al Janaideh, M., Rakheja, S., Su, C. Y. An analytical generalized Prandtl-Ishlinskii model inversion for hysteresis compensation in micropositioning control, IEEE/ASME Transactions on Mechatronics, 16 (4), 734-744, (2011). https://dx.doi.org/10.1109/TMECH.2010.2052366

18 Preisach, F. Uber die magnetische Nachwirkung, Zeitschrift fui hysic, 94 (5-6), 277-302, (1935). https://dx.doi.org/10.1007/BF01349418

19 Mayergoyz, I. Mathematical Models of Hysteresis and their Application, Series in Electromagnetism, Elsevier, New York, (2003). https://dx.doi.org/10.1016/B978-0-12480873-7.50010-4

20 Hughes, D., Wen, J. T. Preisach modeling of piezoceramic and shape memory alloy hysteresis, Smart Materials and Structures, 6 (3), 287-300, (1997). https://dx.doi.org/10.1109/CCA.1995.555909

21 Ge, P., Jouaneh, M. Tracking control of a piezoceramic actuator, IEEE Transactions on Control Systems Technology, 4 (3), 209-216, (1996). https://dx.doi.org/10.1109/87.491195

22 Ge, P., Jouaneh, M. Generalized preisach model for hysteresis nonlinearity of piezoceramic actuators, Precision Engineering, 20 (2), 99-111, (1997). https://dx.doi.org/10.1016/S0141-6359(97)00014-7

23 Robert, G., Damjanovic, D., Setter, N., Turik, A. V. Preisach modeling of piezoelectric nonlinearity in ferroelectric ceramics, Journal of Applied Physics, 89 (9), 50675074, (2001). https://dx.doi.org/10.1063/1.1359166

24 Robert, G., Damjanovic, D., Setter, N. Preisach distribution function approach to piezoelectric nonlinearity and hysteresis, Journal of Applied Physics, 90 (5), 2459-2464, (2001). https://dx.doi.org/10.1063/1.1388855

25 Wilkie, W. K. Low-cost piezocomposite actuator for structural control applications, In SPIE's 7th Annual International Symposium on Smart Structures and Materials, 323334, (2000). https://dx.doi.org/10.1117/12.388175

26 Bilgen, O., Friswell, M. I., Inman, D. J. Theoretical and experimental analysis of hysteresis in piezocomposite airfoils using Preisach model, Journal of Aircraft, 48 (6), 19351947, (2011). https://dx.doi.org/10.2514/1.C031374 